\title{
Review Article \\ The MINOS Experiment: Results and Prospects
}

\author{
J. J. Evans \\ Department of Physics and Astronomy, University of Manchester, Oxford Road, Manchester M13 9PL, UK
}

Correspondence should be addressed to J. J. Evans; justin.evans@hep.manchester.ac.uk

Received 27 June 2013; Accepted 16 September 2013

Academic Editor: Leslie Camilleri

Copyright (c) 2013 J. J. Evans. This is an open access article distributed under the Creative Commons Attribution License, which permits unrestricted use, distribution, and reproduction in any medium, provided the original work is properly cited.

\begin{abstract}
The MINOS experiment has used the world's most powerful neutrino beam to make precision neutrino oscillation measurements. By observing the disappearance of muon neutrinos, MINOS has made the world's most precise measurement of the larger neutrino mass splitting and has measured the neutrino mixing angle $\theta_{23}$. Using a dedicated antineutrino beam, MINOS has made the first direct precision measurements of the corresponding antineutrino parameters. A search for $\nu_{e}$ and $\bar{\nu}_{e}$ appearance has enabled a measurement of the mixing angle $\theta_{13}$. A measurement of the neutral-current interaction rate has confirmed oscillation between three active neutrino flavours. MINOS will continue as MINOS+ in an upgraded beam with higher energy and intensity, allowing precision tests of the three-flavour neutrino oscillation picture, in particular a very sensitive search for the existence of sterile neutrinos.
\end{abstract}

\section{Introduction}

The MINOS experiment, as an idea, was conceived in the late 1990s [1]. This was a very important period in neutrino oscillation physics. For thirty years, results from Homestake [2] and the gallium experiments $[3,4]$, through to a number of atmospheric neutrino detectors [5-10], had shown that neutrinos behaved in an odd fashion, often showing significant deficits from the expected flux, but none had conclusively determined the mechanism responsible. Then, in 1998, Super-Kamiokande [11] proved decisively that muon neutrinos produced in the Earth's atmosphere disappeared as they traveled. Around three years later, the SNO experiment showed conclusively that neutrinos, as they propagated, changed between their three flavours $[12,13]$. This discovery of neutrino flavour change showed that neutrinos had mass and did not conserve lepton number; it was the first, and still the only, observation of physics beyond the standard model.

It was during this period of discovery that the MINOS experiment was proposed, to begin an era of precision measurement of this new phenomenon. The data at the time were well modeled by the theory of neutrino oscillation, in which the rate of oscillation between the three flavours is governed by the differences between the squared neutrino masses, $\Delta m_{21}^{2}$, and $\Delta m_{32}^{2}$. The magnitude of the flavour change is governed by three mixing angles, $\theta_{12}, \theta_{23}$, and $\theta_{13}$, and a $\mathscr{C} \mathscr{P}$-violating phase $\delta$; these parameters form the PMNS rotation matrix [14-16] that relates the neutrino mass eigenstates to the flavour eigenstates. Nature has decreed that the two mass splittings differ by more than an order of magnitude and that one of the mixing angles, $\theta_{13}$, is small. Therefore, oscillation phenomenology can be divided into two distinct regimes: "solar" oscillation driven by $\Delta m_{21}^{2}$ and $\theta_{12}$ and "atmospheric" oscillation driven by $\Delta m_{32}^{2}$ and $\theta_{23}$. MINOS was designed to make precision measurements of the parameters governing the atmospheric oscillation regime; however, it has also played a role in the measurement of $\theta_{13}$ and will, in the future, make sensitive searches for the existence of sterile neutrinos. An important feature of the MINOS design is the ability of the detectors to identify both $\nu_{\mu}$ and $\bar{\nu}_{\mu}$ interactions separately. This has allowed MINOS to make the first direct precision tests that the values of $\Delta m_{32}^{2}$ and $\theta_{23}$ are the same for neutrinos and antineutrinos [17-20].

To achieve its goals, the MINOS experiment uses the world's most powerful neutrino beam, the NuMI beam. To make best use of this beam, the experiment has pioneered a number of techniques associated with the use of the twodetector arrangement over very long baselines, which is now the gold standard for all neutrino oscillation experiments. 


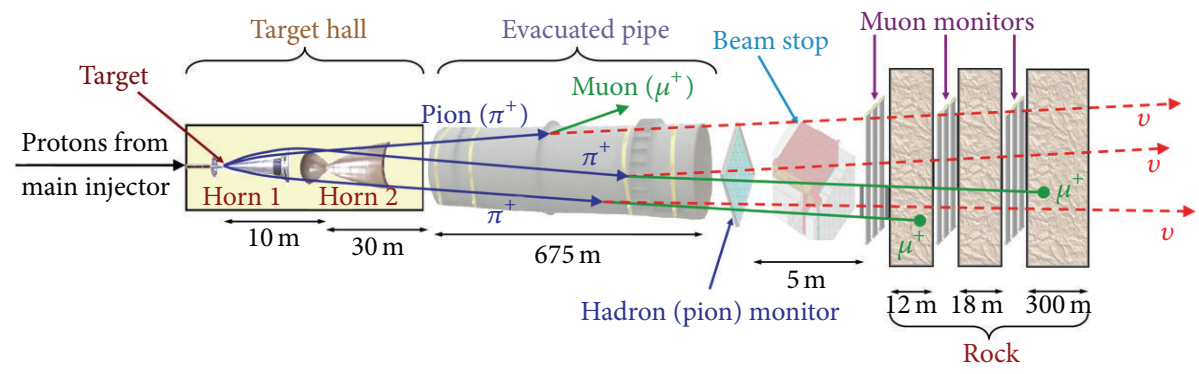

Figure 1: The NuMI beam.

\section{The MINOS Experiment}

The NuMI facility [21] provides MINOS with an intense beam of muon flavoured neutrinos at energies of a few $\mathrm{GeV}$. The atmospheric neutrino mass splitting drives oscillation predominantly between muon and tau flavour neutrinos; the energy dependence of the muon neutrino survival probability is given by

$$
\begin{aligned}
& P\left(v_{\mu} \longrightarrow v_{\mu}\right) \\
& \quad=1-\sin ^{2}(2 \theta) \sin ^{2}\left(\frac{1.27 \Delta m^{2}\left[\mathrm{eV}^{2}\right] L[\mathrm{~km}]}{E[\mathrm{GeV}]}\right)
\end{aligned}
$$

In this two-flavour approximation, $\Delta m^{2}$ is an admixture of $\Delta m_{32}^{2}$ and $\Delta m_{31}^{2} ; \theta$ is also an admixture of the mixing angles but is heavily dominated by $\theta_{23}$. Since MINOS cannot observe the $\nu_{\tau}$ appearance, it is the measurement of this $\nu_{\mu}$ survival probability that is used to determine the parameters $\theta$ and $\Delta m^{2}[20,22-25]$.

A nonzero $\theta_{13}$ causes a small amount of $\nu_{e}$ appearance in the beam, with an energy dependence given by

$$
\begin{aligned}
& P\left(v_{\mu} \longrightarrow v_{e}\right) \\
& \quad \approx \sin ^{2}\left(\theta_{23}\right) \sin ^{2}\left(2 \theta_{13}\right) \sin ^{2}\left(\frac{1.27 \Delta m^{2}\left[\mathrm{eV}^{2}\right] L[\mathrm{~km}]}{E[\mathrm{GeV}]}\right) .
\end{aligned}
$$

MINOS has selected a sample of $\nu_{e}$-enhanced events to make a measurement of $\theta_{13}$ [26-29].

An important signature of neutrino oscillation is that the rate of neutral-current (NC) neutrino interactions is unchanged by the process. The NC interaction is equally sensitive to all three neutrino flavours, so this proves that flavour change is occurring between the three active neutrino flavours. By analysing NC interactions, MINOS has confirmed that oscillation is the correct picture and has shown no evidence that this oscillation includes additional, sterile neutrino flavours [30-32].

The NuMI beam [21], based at Fermilab in Chicago, has run since 2005 and has reached a typical beam power of $350 \mathrm{~kW}$. The Fermilab main injector produces a $10 \mu \mathrm{s}$ pulse of around $3 \times 10^{13}$ protons every $2.2 \mathrm{~s}$. These protons have an energy of $120 \mathrm{GeV}$ and strike a graphite target, as shown in Figure 1. This target has a length of 2.0 nuclear interaction lengths and consists of a series of forty-seven $2 \mathrm{~cm}$ long graphite fins, separated by $0.3 \mathrm{~mm}$. A shower of hadrons is produced at the target, consisting primarily of pions with a significant kaon component at higher energies. These hadrons pass through two parabolic, magnetic horns which focus either positive or negative hadrons depending on the direction of the electric current through the horns. The focused hadrons pass down a $675 \mathrm{~m}$ long, helium filled pipe, in which they decay to produce a beam of predominantly muon flavoured neutrinos, with a small electron neutrino component from the decays of muons and kaons.

Figure 2 shows the composition of the NuMI beam. With the horns configured to focus positive hadrons, the spectrum of charged current (CC) interactions observed in the MINOS near detector at Fermilab consists of $91.7 \% \nu_{\mu}$, $7.0 \% \bar{\nu}_{\mu}$, and $1.3 \% \nu_{e}$ and $\bar{\nu}_{e}$. With the horns focusing negative hadrons, the observed CC interactions consist of $39.9 \% \bar{\nu}_{\mu}$, $58.1 \% \nu_{\mu}$, and $2.0 \% \nu_{e}$ and $\bar{\nu}_{e}$. The significant difference in composition and event rate between these two configurations arises mainly from the fact that the $\bar{\nu}_{\mu}$ interaction crosssection is approximately a factor of two lower than the $\nu_{\mu}$ interaction cross-section.

The neutrino beam peaks at an energy of close to $3 \mathrm{GeV}$. However, the current through the focusing horns and the relative positions of the horns and target are variable, allowing the energy of the beam peak to be varied to as high as $10 \mathrm{GeV}$. This feature has enabled MINOS to study and understand the beam in detail [33], improving the simulation of the beam beyond the raw Fluka [34] and GEANT $[35,36]$ Monte Carlos and significantly reducing the systematic uncertainty from the modeling of the neutrino flux.

A total of $10.56 \times 10^{20}$ protons on target of beam data has been analysed in the neutrino-dominated beam mode. This data corresponds to the beam configuration shown in Figure 2, with the energy spectrum peaking at $3 \mathrm{GeV}$, and is referred to as the low energy beam configuration. Additional $0.15 \times 10^{20}$ protons on target of data with a $10 \mathrm{GeV}$ beam peak have also been used. In the antineutrino-enhanced beam mode, a total of $3.36 \times 10^{20}$ protons on target of beam data have been analysed. These are the exposures used for all analyses presented in this paper except where otherwise stated, and they were obtained between May 2005 and April 2012.

The two MINOS detectors [37] are steel-scintillator calorimeters, shown in Figure 3. They consist of planes of 


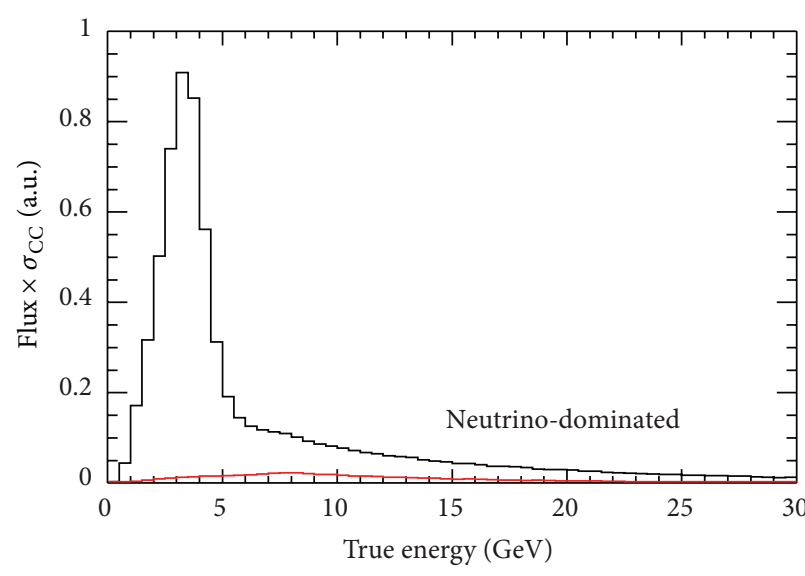

Near detector simulated Low energy beam $-v_{\mu}$ spectrum

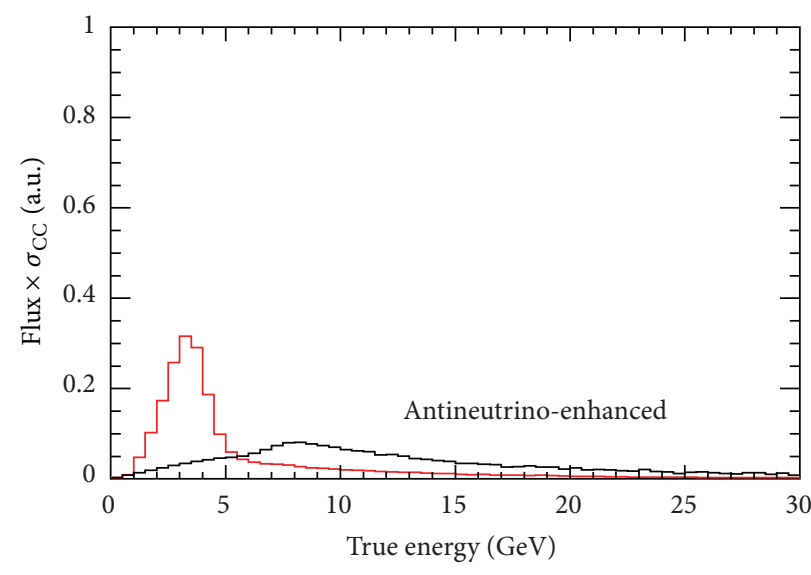

Near detector simulated Low energy beam

$-v_{\mu}$ spectrum

Figure 2: The composition of the NuMI beam, when configured to produce (a) a neutrino-dominated beam and (b) an antineutrino-enhanced beam. The figures show the rate of charged current neutrino interactions observed in the MINOS near detector.

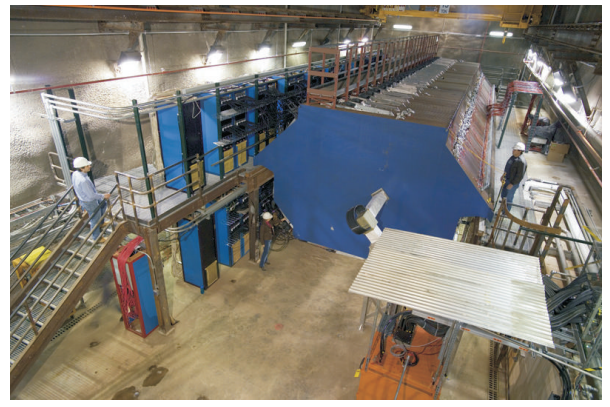

(a)

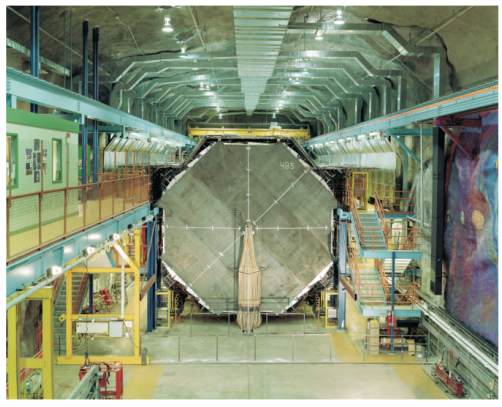

(b)

FIgURE 3: The MINOS detectors. (a) the near detector at Fermilab; (b) the far detector at the Soudan Underground Laboratory.

inch-thick steel, interleaved with planes of $1 \mathrm{~cm}$ thick plastic scintillator. The scintillator planes are divided into $4 \mathrm{~cm}$ wide strips, as shown in Figure 4. Along the centre of each strip, a wavelength shifting fibre collects the scintillation light, shifts it to green wavelengths, and takes it out to a photomultiplier tube. Any charged particles passing through the detector deposit their energy to produce light; the pattern of these deposits allows the topology of the neutrino interaction to be reconstructed. The scintillator strips are aligned orthogonally on adjacent detector planes, to allow three-dimensional reconstruction. The detectors are magnetised to around $1.3 \mathrm{~T}$, allowing the charge of particles to be identified.

The smaller of the two detectors, the Near Detector (ND), sits at Fermilab, $1.04 \mathrm{~km}$ from the target. With a mass of $0.98 \mathrm{kton}$, it measures the energy spectra of the neutrinos before oscillation. The far detector is located at the Soudan Underground Laboratory in northern Minnesota, $705 \mathrm{~m}$ underground and $735 \mathrm{~km}$ from the target. With a mass of $5.4 \mathrm{kton}$, it again measures the neutrino energy spectra, seeing the appearance and disappearance of neutrinos due to oscillation.

This two-detector arrangement, previously used over distances of around $1 \mathrm{~km}$ by experiments such as CCFR, CDHS, and CHARM [38-40] and then over $250 \mathrm{~km}$ by K2K [41], is very powerful in reducing systematic uncertainties. Neutrino physics is beset with uncertainty: in particular, interaction cross-sections are unknown to many tens of per cent, and neutrino fluxes can be mismodeled by similar amounts. However, these uncertainties affect both the near and far detectors in very similar ways. Thus, when a ratio is taken of the energy spectra measured in the two detectors, a cancellation occurs and the effects of the uncertainties are greatly reduced. As an indication of how well this works, despite the uncertainties of tens of per cent in the simulated event rate in the detectors, once the near to far detector ratio is taken, the normalization is known to $1.6 \%$; this $1.6 \%$ is dominated by the uncertainty in the relative efficiency of the event reconstruction algorithms between the two detectors. 


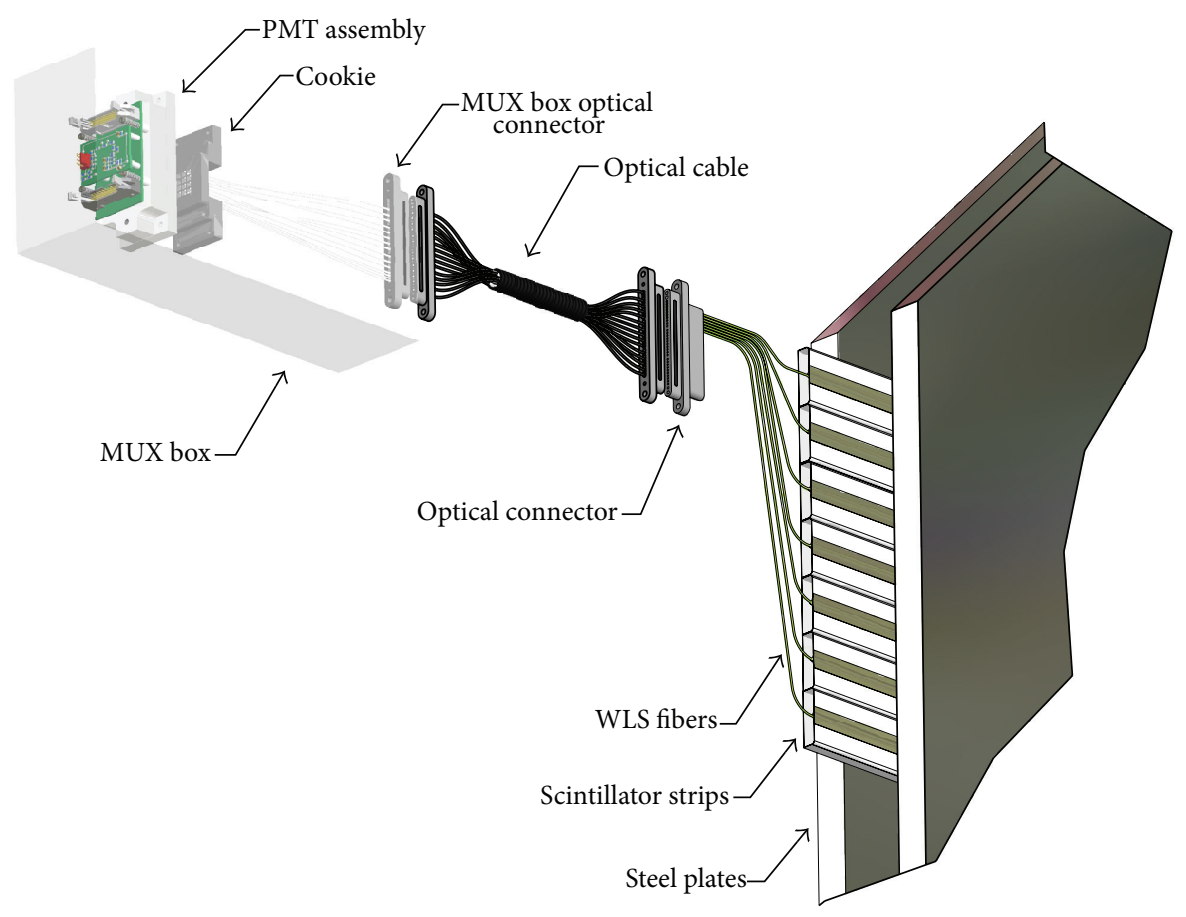

FIGURE 4: A MINOS detector plane.

The MINOS Far Detector is also a very effective detector of neutrinos produced in the atmosphere. Since it was switched on in 2003, it has recorded 37.9 kton-years of data, recording 2072 candidate neutrino interactions that have been included into the analyses of the beam data to improve the precision of the oscillation parameter measurements [20, 42-44].

\section{Neutrino Interactions in the MINOS Detectors}

Three types of neutrino interaction, shown in Figure 5, are of interest to MINOS. Muon neutrinos and antineutrinos interact through the CC process:

$$
v_{\mu}\left(\bar{\nu}_{\mu}\right)+X \longrightarrow \mu^{-(+)}+X^{\prime} .
$$

The cascade of hadrons, $X^{\prime}$, produces a diffuse shower of energy deposits near the interaction vertex. The muon produces a long track that curves in the magnetic field the direction of curvature identifying the incoming neutrino as a $\nu_{\mu}$ or a $\bar{\nu}_{\mu}$.

All active neutrino flavours undergo NC interactions through the process:

$$
v+X \longrightarrow v+X^{\prime} .
$$

Only the hadronic cascade is observed, producing a diffuse shower of energy deposits.

Finally, electron neutrinos undergo CC interactions through the process:

$$
v_{e}+X \longrightarrow e^{-}+X^{\prime}
$$

The electron gives rise to an electromagnetic shower, which produces a much denser, more compact shower of energy deposits.

The energy of the neutrino is determined by summing the energies of the shower and any muon track. The muon energy is determined from the length of stopping tracks, leading to a resolution of around 5\%, and from the curvature in the magnetic field for tracks that exit the detector, leading to a resolution of around $10 \%$. For NC and $v_{e}$ CC interactions, the energy of the shower is determined through calorimetry. The calorimetric energy resolution for hadronic showers is around 55\%/ $\sqrt{\text { energy }}[45]$ and for electromagnetic showers $20 \% / \sqrt{\text { energy }}[46]$. For $v_{\mu}$ CC interactions, a more sophisticated approach is used to improve the resolution of hadronic shower energy measurement [47]. For low energy showers (of a few $\mathrm{GeV}$ or below), significant additional information is held in the topology of the shower. Three event characteristics are used: the calorimetric energy deposit within $1 \mathrm{~m}$ of the interaction vertex, the sum of the calorimetric energy in the two largest showers in the event, and the physical length of the largest shower. These variables are input into a $k$-nearestneighbour algorithm [48], which finds the best matches from a library of simulated events and uses these to estimate the hadronic energy. This improves the shower energy resolution from $55 \%$ to $43 \%$ for showers between $1.0 \mathrm{GeV}$ and $1.5 \mathrm{GeV}$.

3.1. Selection of Charged-Current $v_{\mu}$ and $\bar{v}_{\mu}$ Interactions. To make a measurement of $P\left(\nu_{\mu} \rightarrow \nu_{\mu}\right)$, it is necessary to select a pure sample of $\nu_{\mu}$ CC interactions. This is achieved by selecting events with a clear muon track. The main loss in efficiency comes from events with a high inelasticity in which a short muon track is hidden in a large hadronic cascade. 


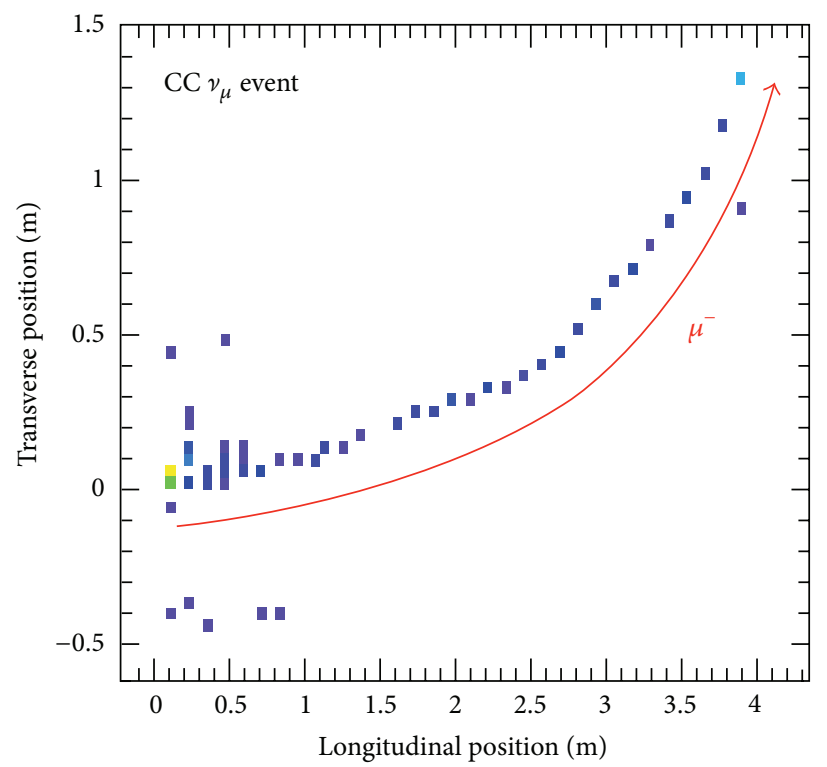

(a)

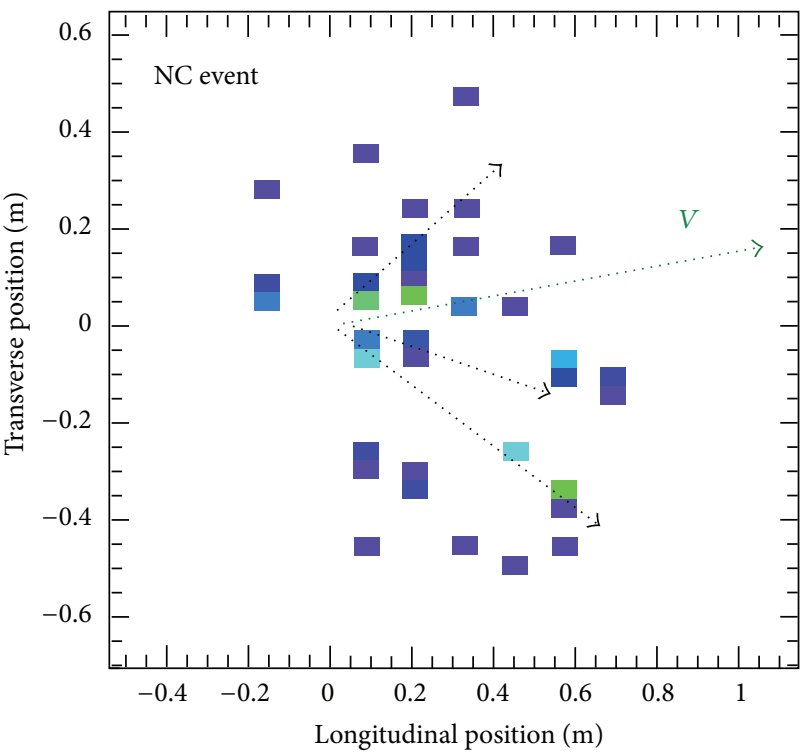

(b)

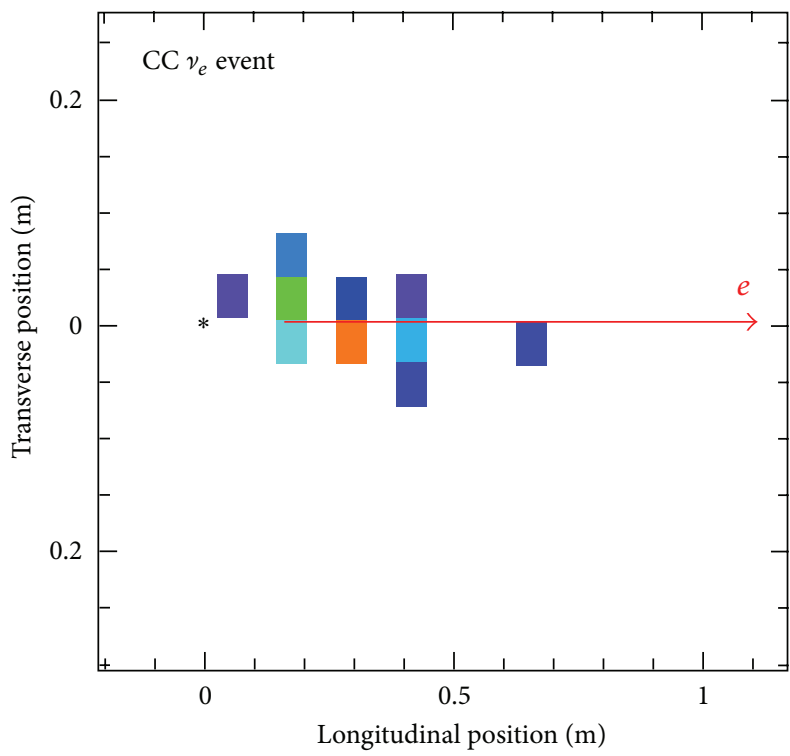

(c)

FIgURE 5: Neutrino interaction topologies observed in the MINOS detectors. (a) A CC $v_{\mu}$ interaction. (b) A NC interaction. (c) A CC $v_{e}$ interaction. Each coloured rectangle represents an excited scintillator strip, the colour indicating the amount of light: purple and blue are low light levels, through to orange and red for the highest light levels.

The main background occurs at low energies and consists of small cascades from NC interactions in which a low energy hadron, such as a proton or a charged pion, exhibits a tracklike topology that mimics a low energy muon. Four variables are constructed that discriminate between muons tracks, which are typically long and show a constant energy deposition along the length and spurious hadronic tracks, which are typically shorter and show greater fluctuations in the energy deposition. These variables are the event length, the average energy deposited per scintillator plane along the track, the transverse energy deposition profile, and the fluctuation of the energy deposition along the track. These variables are input into a $k$-nearest-neighbour algorithm, which calculates a single discrimination variable, shown in Figure 6 [49]. Events for which this variable is greater than 0.3 are selected as CC $v_{\mu}$ interactions, yielding a sample with a total efficiency of $90 \%$; below $2 \mathrm{GeV}$, the NC contamination is $6.5 \%$. The efficiency and contamination are energy dependent; this full energy dependence is shown in Figure 6.

The CC interactions of $\nu_{\mu}$ and $\bar{\nu}_{\mu}$ result in very similar topologies; the $k$-nearest-neighbour discriminant is therefore used in the same way in both the neutrino-dominated and 


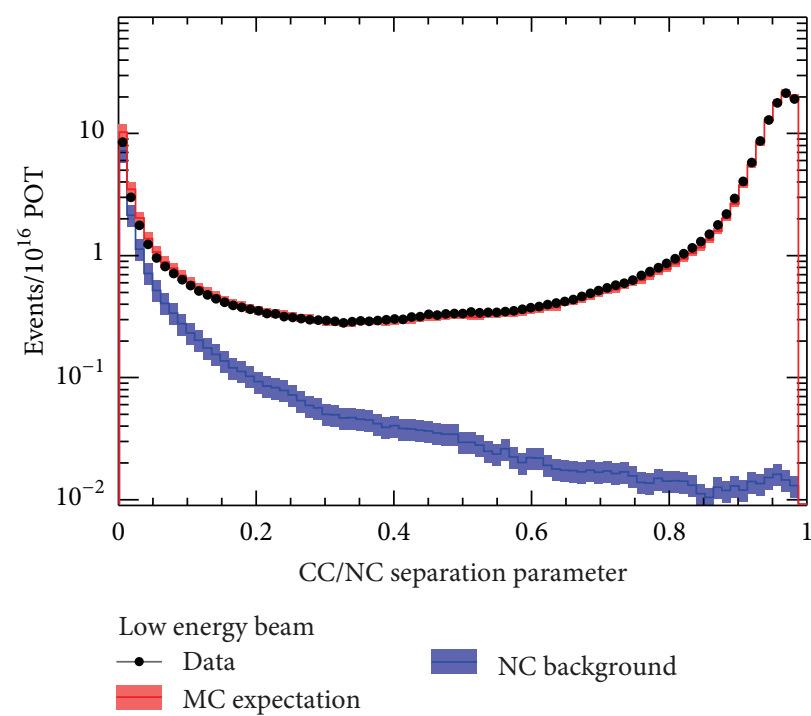

(a)

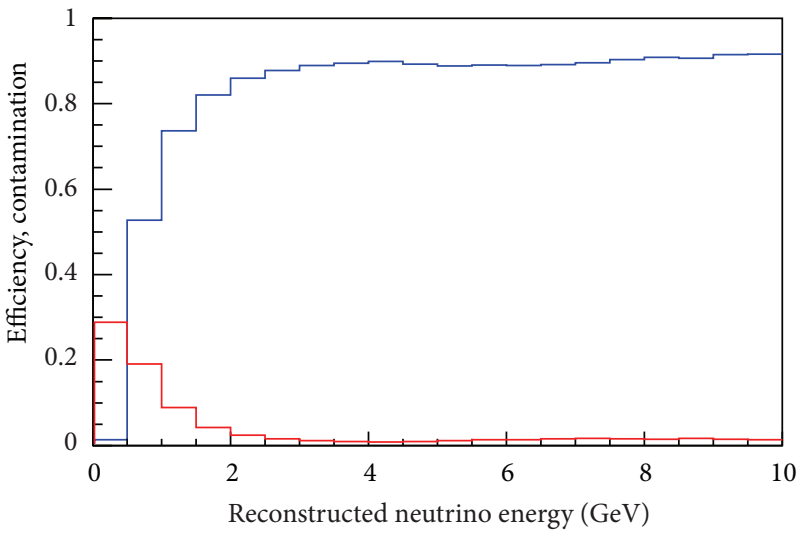

Far detector fiducial only - CC selection efficiency - NC contamination

(b)

FIGURE 6: (a) The discrimination variable used to separate $v_{\mu}$ CC interactions from hadronic backgrounds. Events with a parameter value greater than 0.3 are selected as $v_{\mu}$ CC interactions. (b) The efficiency and background contamination of the selected $\nu_{\mu}$ CC sample in the far detector.

antineutrino-enhanced beams. When performing a direct measurement of the antineutrino oscillation parameters, an additional selection cut is made, requiring the charge of the muon track to be positive. This uses the direction of curvature of the muon as measured by a Kalman Filter algorithm [50]. A further sample of $\bar{\nu}_{\mu}$ CC interactions is obtained from the 7\% $\bar{\nu}_{\mu}$ component in the neutrino-dominated beam. This sample contains a significant background of $\nu_{\mu}$ events in which a $\mu^{-}$ has been identified with the incorrect charge, often at low energies where the muon undergoes significant scattering. Therefore a much stricter set of selection criteria are applied to purify this $\bar{v}_{\mu}$ sample [18].

3.2. Selection of Charged-Current $v_{e}$ Interactions. The selection of $v_{e}$ CC interactions focuses on identifying the dense showers from the electromagnetic interaction of the electron, rather than the much more diffuse hadronic showers. The primary background comes from purely hadronic showers which can have a denser than average energy deposit, particularly in the presence of a neutral pion decaying to photons. Once a set of shower-like events in the signal region of $1-8 \mathrm{GeV}$ has been obtained, a pattern matching approach, called library event matching, is used to identify the interactions most likely to be $v_{e}$ CC $[51,52]$. Each event in the data is compared to a library of $5 \times 10^{7}$ simulated signal and background events; its similarity to the library events is quantified by comparing the pattern of energy deposits in each scintillator strip excited by the shower, where the energy deposit is quantified by the charge recorded on the photomultiplier tube. For an arbitrary energy deposit, the mean expected charge on a photomultiplier tube will be some value $\lambda$. The probability of observing an amount of charge $n$ is then a Poisson distribution, $P(n \mid \lambda)$. The likelihood, $\mathscr{L}$, of a data event corresponding to the same physical shower topology as a simulated library event can therefore be calculated as

$$
\log \mathscr{L}=\sum_{i=1}^{N_{\text {strips }}} \log \left[\int_{0}^{\infty} P\left(n_{\text {data }}^{i} \mid \lambda\right) P\left(n_{\text {lib }}^{i} \mid \lambda\right) \mathrm{d} \lambda\right]
$$

where $i$ represents the $i$ th scintillator strip in the shower. Using this definition of the likelihood, the 50 library events are identified that best match the data event. Three quantities are calculated from this set of 50 best-matching library events: the fraction of the events that are true $v_{e}$ CC events, the average inelasticity of the true $v_{e} \mathrm{CC}$ events, and the average fraction of charge that overlaps between the data event and each $v_{e}$ CC library event. These three quantities are input to a neural network, which calculates a classification variable shown in Figure 7. Events with a classification variable value above 0.6 are selected for analysis; this value was chosen to maximise the sensitivity to $v_{e}$ and $\bar{v}_{e}$ appearance.

The efficiency of the $v_{e}$ CC selection is estimated from the data, rather than relying totally on the simulation. To obtain a pure sample of true hadronic showers, a sample of well-identified $v_{\mu}$ CC events is selected, and the energy depositions corresponding to the muon track are removed [53]. The simulated energy depositions of an electron are then inserted [54], providing a realistic sample of $v_{e}$ CC events. Using this method, the $v_{e}$ CC identification efficiency is found to be $(57.4 \pm 2.8) \%$ in the neutrino-dominated beam and $(63.3 \pm 3.1) \%$ in the antineutrino-enhanced beam.

3.3. Selection of Neutral-Current Interactions. The signal of an NC interaction is a diffuse hadronic shower. $v_{\mu}$ CC interactions also produce hadronic showers, and if the inelasticity is high, the tell-tale muon track may not visibly extend past the shower. To purify a sample of NC interactions, a simple 


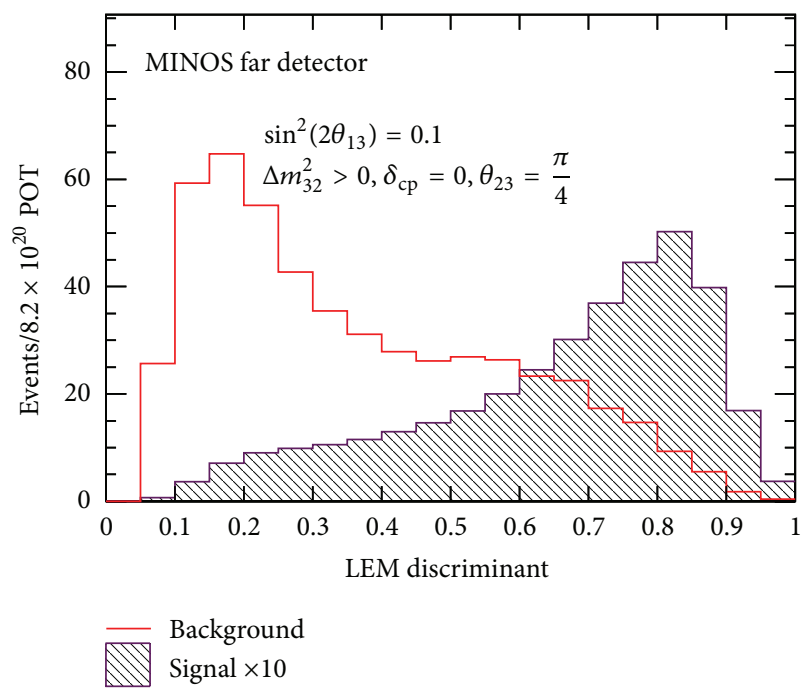

(a)

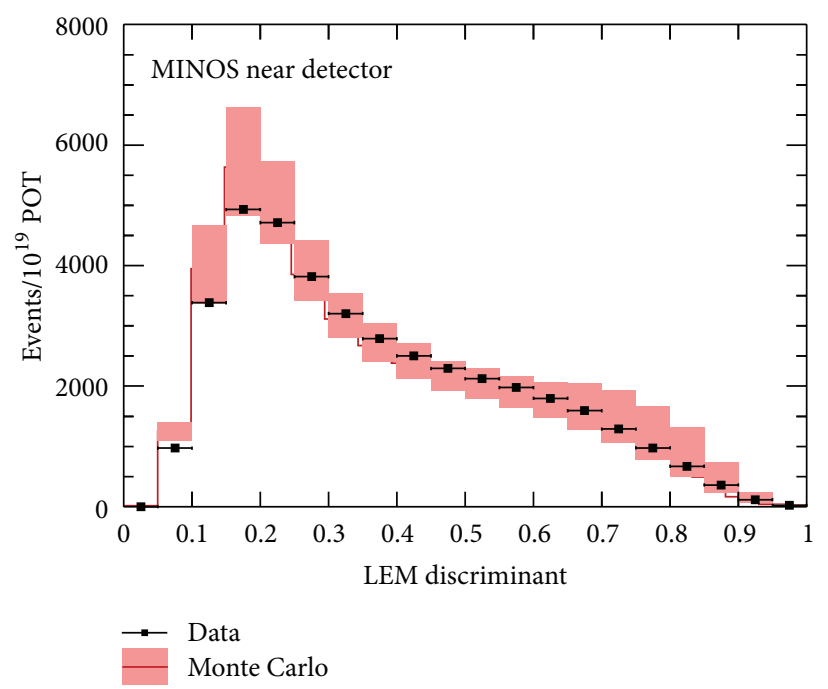

(b)

FIGURE 7: (a) The library event matching discriminant, showing the expected distribution for background and CC $\nu_{e}$ signal events in the far detector, in the neutrino-dominated beam. Note that the signal, simulated for $\sin ^{2}\left(2 \theta_{13}\right)=0.1, \delta=0$, and a normal mass hierarchy, has been scaled up by a factor of ten for visibility. (b) The same discriminant as observed in the near detector, compared with the simulated expectation.

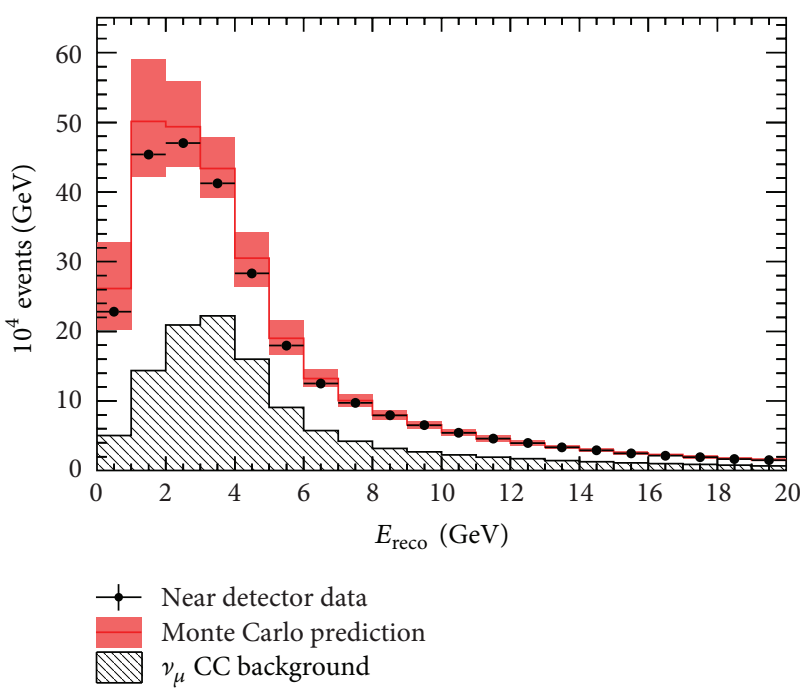

Figure 8: The sample of events identified as NC interactions in the near detector.

cut-based approach is taken [55]: events are classified as NC-like if the event contains no reconstructed track or if the track extends no more than six planes past the end of the shower. The resulting distribution of NC interactions in the near detector is shown in Figure 8. The NC identification efficiency is $89 \%$, with $61 \%$ purity. This selection will identify $97 \%$ of $\nu_{e}$ CC interactions as NC events; therefore, an analysis of NC interactions in the FD must account for the $v_{e}$ appearance caused by a nonzero $\theta_{13}$.

3.4. Selection of Atmospheric Neutrinos. Atmospheric neutrino interactions are selected out of any activity seen in the FD outside of the $10 \mu$ s periods when the NuMI beam is active [44]. The oscillation signal is contained in the $v_{\mu}$ CC interactions, and, as with the beam-induced interactions, these are identified by the presence of a muon track. The FD began taking data with atmospheric neutrinos in July 2003, two years before the NuMI beam began running.

The FD has a single-hit timing resolution of $2.5 \mathrm{~ns}$. This timing information is used to determine the direction in which the detector activity is traveling. Any downwards traveling activity is required to begin well inside the detector, to eliminate cosmic muons entering from above. All upwards or horizontally traveling activity is almost certain to be neutrino-induced, since no other particle can survive through the many kilometres of rock. All activity with a zenith angle of $\cos \theta_{z}<0.14$ is defined as horizontal or downgoing; this corresponds to an overburden of at least $14 \mathrm{~km}$ water equivalent.

From this sample of neutrino-induced activity, all events with a track crossing at least eight planes are designated track-like; all events with only shower-like activity crossing at least four planes are designated shower-like. These track-like and shower-like samples are used in the neutrino oscillation measurements. The track-like sample contains the oscillation signal of $\nu_{\mu}$ disappearance. The shower-like sample contains mainly NC interactions and $\nu_{e}$ and $\bar{\nu}_{e}$ CC interactions; it shows little oscillation signal but is very important for setting the normalization of the atmospheric neutrino flux.

\section{Muon Neutrino and Antineutrino Disappearance}

The atmospheric oscillation parameters, $\left|\Delta m^{2}\right|$ and $\sin ^{2}(2 \theta)$, are measured by observing and fitting the energy dependence of $\nu_{\mu}$ and $\bar{\nu}_{\mu}$ disappearance. To minimise the impact of 


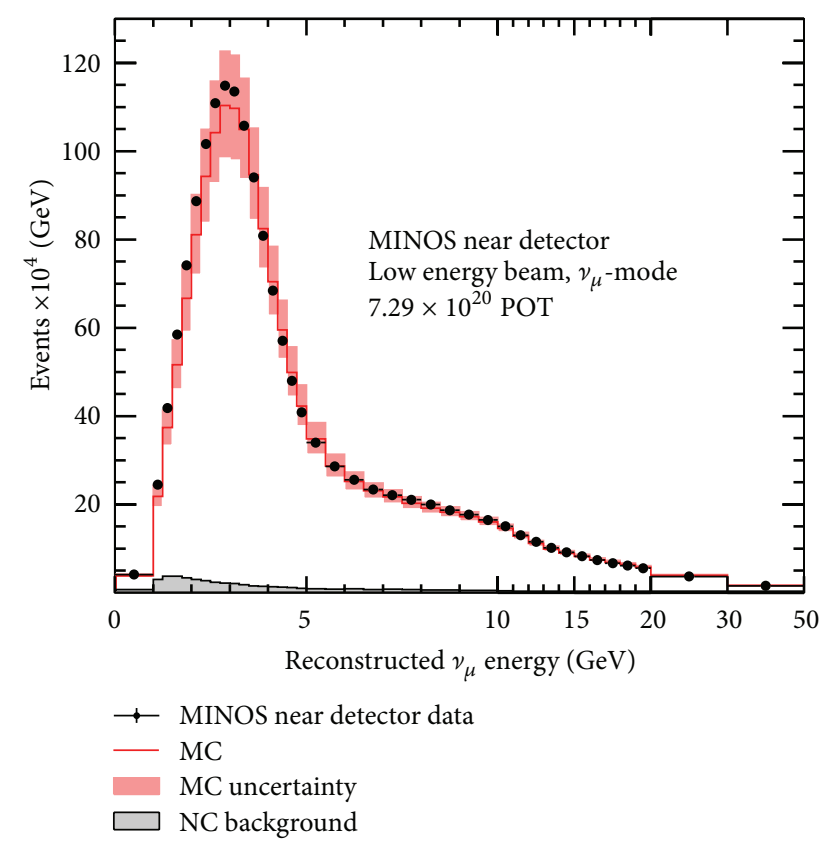

FIGURE 9: The energy spectrum of $\nu_{\mu}$ CC interactions observed in the ND, compared to the simulation.

systematic uncertainties, the energy spectra of the $\nu_{\mu}$ and $\bar{\nu}_{\mu}$ CC interactions observed in the ND (shown in Figure 9 for the neutrino-dominated beam) are used to predict the spectrum at the FD, in the absence of oscillation $[23,56]$. The neutrino energy spectra at the ND and FD are not identical: the ND subtends a relatively large angle to the beam, so for each pion or kaon a range of decay angles can produce a neutrino that passes through the detector, corresponding to a range of neutrino energies. However, the FD is effectively a point when viewed from the neutrino production location, so a single decay angle for each hadron, therefore a single neutrino energy, contributes to the flux. To take this difference into account, the hadron-decay kinematics are encoded into a beam transfer matrix that converts the observed ND flux into a predicted FD flux. Once the ND data has been used in this way, the most important systematic uncertainties are those that can affect the two detectors differently, primarily reconstruction efficiencies and miscalibrations of the neutrino energy measurement in the detectors [57]. These uncertainties are included in the fit that extracts the oscillation parameters [58]. The uncertainty on the reconstruction efficiency is modeled as a $1.6 \%$ uncertainty on the relative rate of events between the ND and FD. The uncertainty on the measurement of muon energy has two components that are fully correlated between the detectors: a $2 \%$ uncertainty on energies measured from range and a $3 \%$ uncertainty on energies measured from the curvature in the magnetic field. The uncertainty in the hadronic energy measurements also has two components. An uncertainty arising from shower modeling uncertainties and calibration is fully correlated between the detectors and is parameterized as $\left(6.6+3.5 e^{E_{\text {shw }} / 1.4 \mathrm{GeV}}\right) \%$. The second component is uncorrelated between the detectors and is $1.9 \%$ in the ND and $1.1 \%$ in the FD; this is dominated by calibration uncertainties.

The top row of Figure 10 shows the predicted spectra of $\nu_{\mu}$ and $\bar{\nu}_{\mu}$ CC interactions from the neutrino-dominated and antineutrino-enhanced beams at the FD, along with the data. In the neutrino-dominated beam, an additional sample is used, consisting of neutrinos interacting outside the fiducial volume of the detector and in the rock surrounding the detector $[59,60]$. This nonfiducial sample consists mainly of high energy neutrinos and has significantly lower resolution as not all the energy is contained in the detector; however, it does contain some oscillation information. In total, 8,100 reconstructed neutrino events are used in the analysis; without oscillations, 9,471 would be expected. In all samples, a clear, energy-dependent deficit of $\nu_{\mu}$ and $\bar{\nu}_{\mu}$ interactions is observed. The ratio of the data to the expectation for the $\nu_{\mu}$ interactions in the neutrino-dominated beam is shown in Figure 11. This ratio shows the "dip and rise" energy dependence of the deficit, which is characteristic of oscillation and described by (1).

The bottom row of Figure 10 shows the spectra of atmospheric $\nu_{\mu}$ and $\bar{\nu}_{\mu}$ CC interactions, as a function of $L / E$, where $L$ is the distance traveled by the neutrino and $E$ is its energy. The atmospheric neutrino events are divided into $\nu_{\mu}$ and $\bar{\nu}_{\mu}$ interactions according to the direction of curvature of the muon and separated into samples depending on whether or not the interaction vertex is contained in the detector.

All the observed $\nu_{\mu}$ and $\bar{\nu}_{\mu}$ CC interactions are fit according to the two-flavour model of (1), under the assumption that neutrinos and antineutrinos have the same oscillation parameters. The resulting measurement of $\left|\Delta m^{2}\right|$ and $\sin ^{2}(2 \theta)$ is shown in Figure 12. The fit yields $\left|\Delta m^{2}\right|=\left(2.41_{-0.10}^{+0.09}\right) \times$ $10^{-3} \mathrm{eV}^{2}$ and $\sin ^{2}(2 \theta)=0.950_{-0.036}^{+0.035}$, disfavouring maximal mixing at the $86 \%$ confidence level. Figure 12 compares this measurement to those from Super-Kamiokande [61] and T2K [62]. The MINOS measurement is the most precise determination of $\left|\Delta m^{2}\right|$, and all measurements of $\sin ^{2}(2 \theta)$ are consistent.

4.1. Muon Antineutrino Disappearance. In the standard model of neutrino oscillation, neutrinos and antineutrinos obey the same parameters, with $\mathscr{C} \mathscr{P} \mathscr{T}$ symmetry requiring that the masses of particles and antiparticles are identical. The most sensitive test of this symmetry in other sectors is from the kaon system [63]. The data from the antineutrinoenhanced beam and the interaction of atmospheric antineutrinos enables the first direct comparison of the neutrino and antineutrino oscillation parameters in the atmospheric region. This comparison provides a limit on nonstandard interactions with the matter being passed through by the neutrino beam [64-70].

Figure 10 showed the energy spectra of $\bar{\nu}_{\mu}$ interactions observed in the FD. These spectra can be fit in the twoflavour model of (1), allowing the antineutrino oscillation parameters to differ from those for neutrinos. This fit yields the antineutrino parameter measurement shown in Figure 13: $\left|\Delta \bar{m}^{2}\right|=\left(2.50_{-0.25}^{+0.23}\right) \times 10^{-3} \mathrm{eV}^{2}$ and $\sin ^{2}(2 \bar{\theta})=0.97_{-0.08}^{+0.03}$. This is in excellent agreement with the parameters measured 

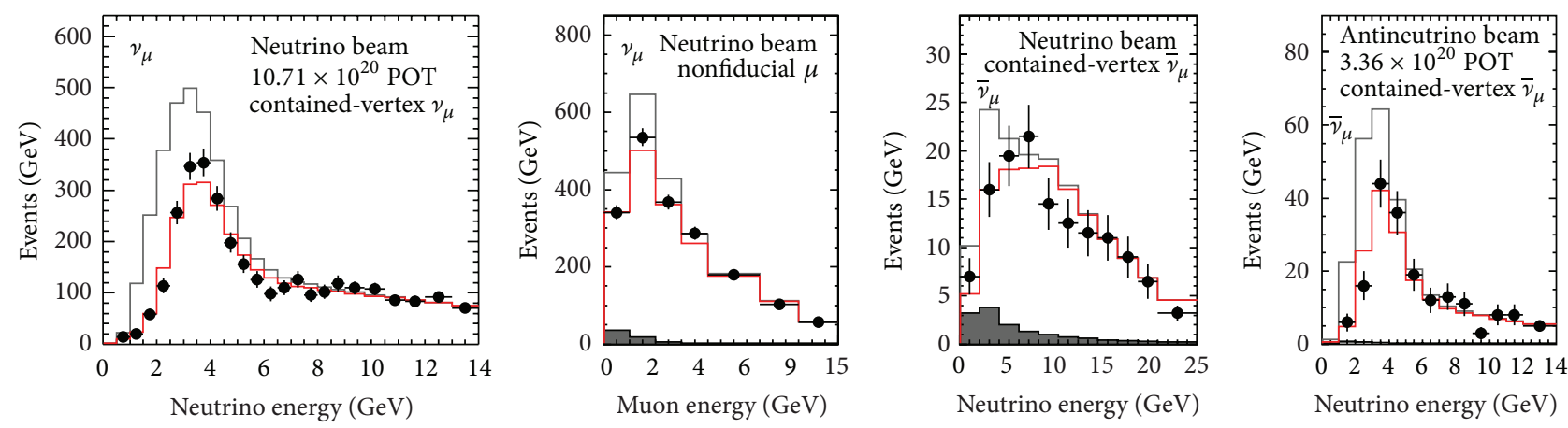

(a)
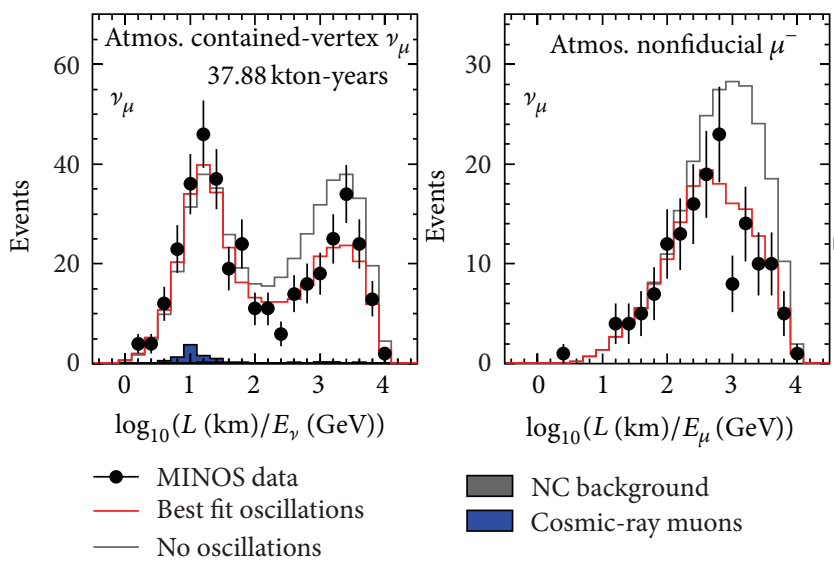
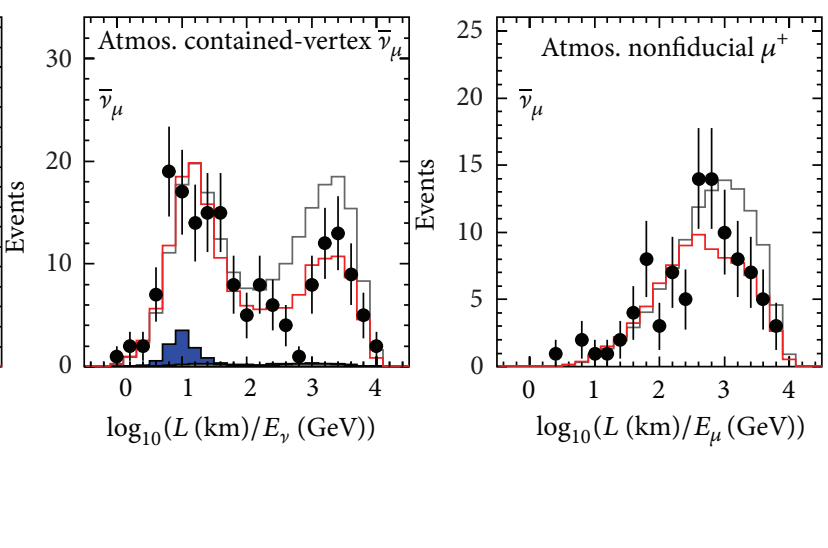

(b)

FIGURE 10: The energy spectra of $\nu_{\mu}$ and $\bar{\nu}_{\mu}$ CC interactions observed at the FD, compared to the expectation with and without oscillation. (a) shows beam-induced neutrinos; (b) shows atmospheric neutrinos.

with neutrinos alone (the red line in Figure 13). It should be noted that the first MINOS measurement of the antineutrino oscillation parameters released in 2010 [17] yielded a notable tension between the $v$ and $\bar{v}$ oscillation parameters, which were in agreement only at the $2.0 \%$ confidence level. This tension was shown to be a statistical fluctuation as the addition of further data brought the $\nu$ and $\bar{\nu}$ parameters measurements into good agreement $[19,20]$.

\section{Electron Neutrino and Antineutrino Appearance}

A search for $\nu_{e}$ and $\bar{\nu}_{e}$ appearance in the $\nu_{\mu}$ and $\bar{\nu}_{\mu}$ beams enables a measurement of the mixing angle $\theta_{13}$. It is critical to know the level of background to the $\nu_{e}$ sample in the FD. The energy spectrum of background events measured in the ND is used to predict the spectrum expected in the FD. However, the background consists of three components: NC interactions, CC $\nu_{\mu}$ and $\bar{\nu}_{\mu}$ interactions, and the intrinsic $\nu_{e}$ component in the beam. The relative contribution between the ND and FD is different for all of these components, since they are affected differently by oscillation, and the kinematics of the production in the beam are different. Therefore, each background must be individually measured. The NuMI beam can be configured to produce neutrino beams of varying energy, by altering the current passing through the magnetic horns and changing the relative positions of the target and horns. Between these different beam configurations, the relative contributions of the three background components change in a well-understood way, as shown in Figure 15. By comparing the ND data to the simulation in the three different beam configurations shown in the figure, the contributions of the three background components can be extracted [71].

Using the data-driven background extraction procedure, a total of 127.7 background events are expected at the FD in the neutrino-dominated beam and 17.5 events in the antineutrino-enhanced beam. In the data, 152 and 20 events are observed, respectively. Figure 16 shows the energy spectra of these events, divided into bins of the library event matching discriminant variable. Although CC $\nu_{e}$ and $\bar{\nu}_{e}$ events cannot be separated on an event-by-event basis, the change in the relative numbers of neutrino and antineutrino interactions between the neutrino-dominated and antineutrinoenhanced beams is well known. This allows separate limits to be placed on the rates of $\nu_{\mu} \rightarrow \nu_{e}$ and $\bar{\nu}_{\mu} \rightarrow \bar{\nu}_{e}$ transitions, on a statistical basis. 


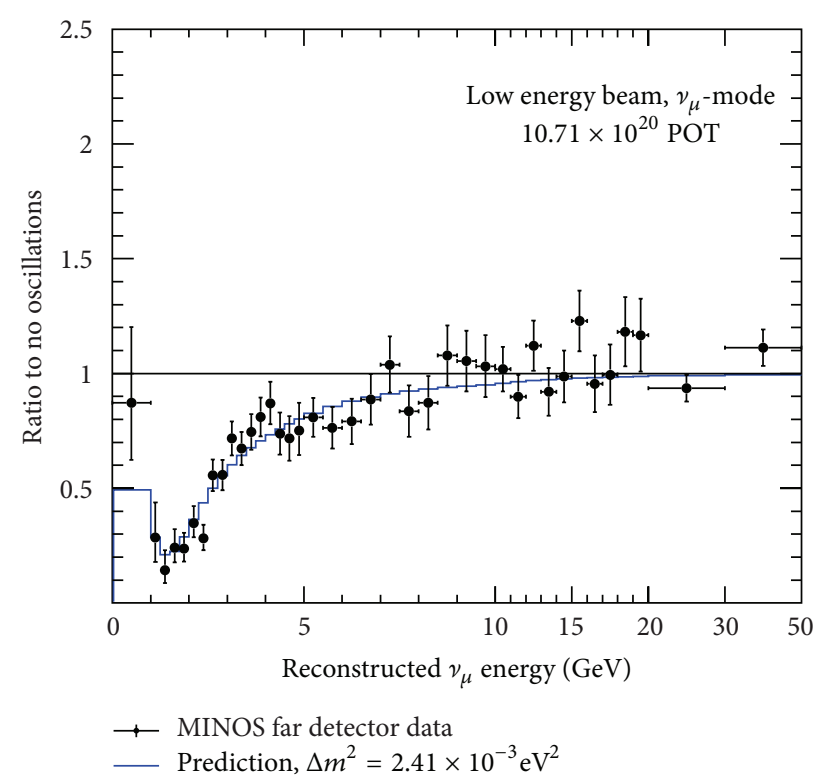

Figure 11: The ratio of the observed $\nu_{\mu}$ energy spectrum to the expectation in the case of no oscillation, in the neutrino-dominated beam. The black points show the data; the blue line shows the best fit to the data.

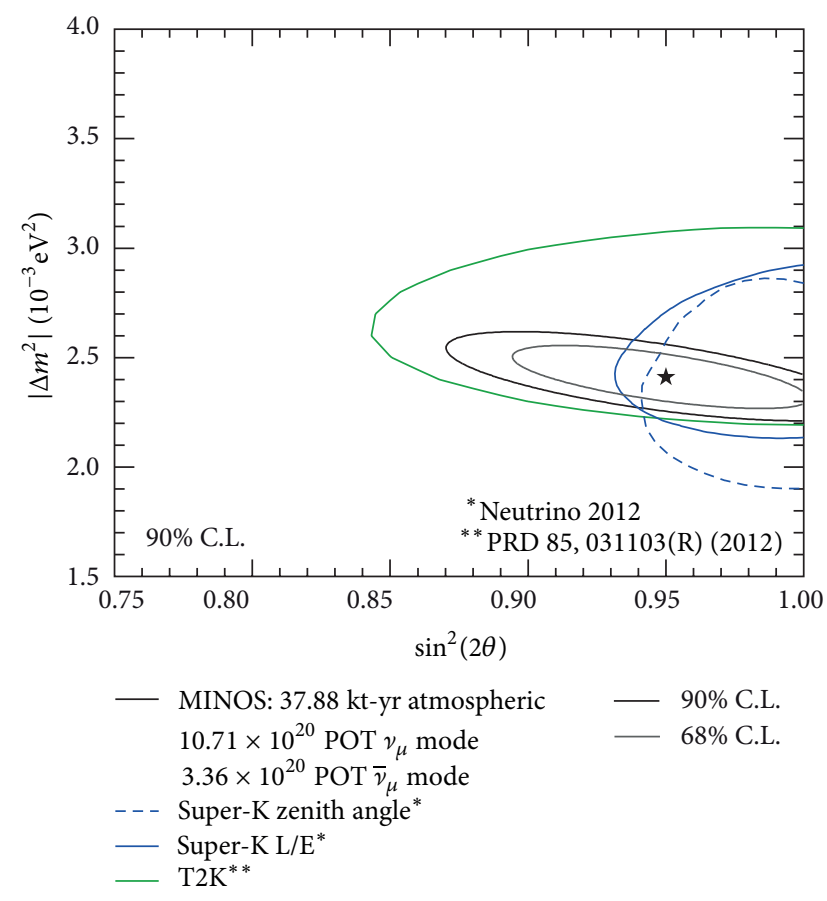

FIGURE 12: The allowed regions for the atmospheric oscillation parameters $\left|\Delta m^{2}\right|$ and $\sin ^{2}(2 \theta)$, assuming identical neutrino and antineutrino oscillation parameters. The MINOS result is compared to measurements from Super-Kamiokande [61] and T2K [62].

The data are fit to extract a measurement of $\theta_{13}$. The resulting measurement is shown in Figure 17. The measured value of $\theta_{13}$ depends on the $\mathscr{C} \mathscr{P}$ violating phase $\delta$, which directly affects the $\nu_{e}$ and $\bar{\nu}_{e}$ appearance probabilities, and the mass hierarchy, which affects the appearance probabilities through the interactions of the neutrinos with the matter in

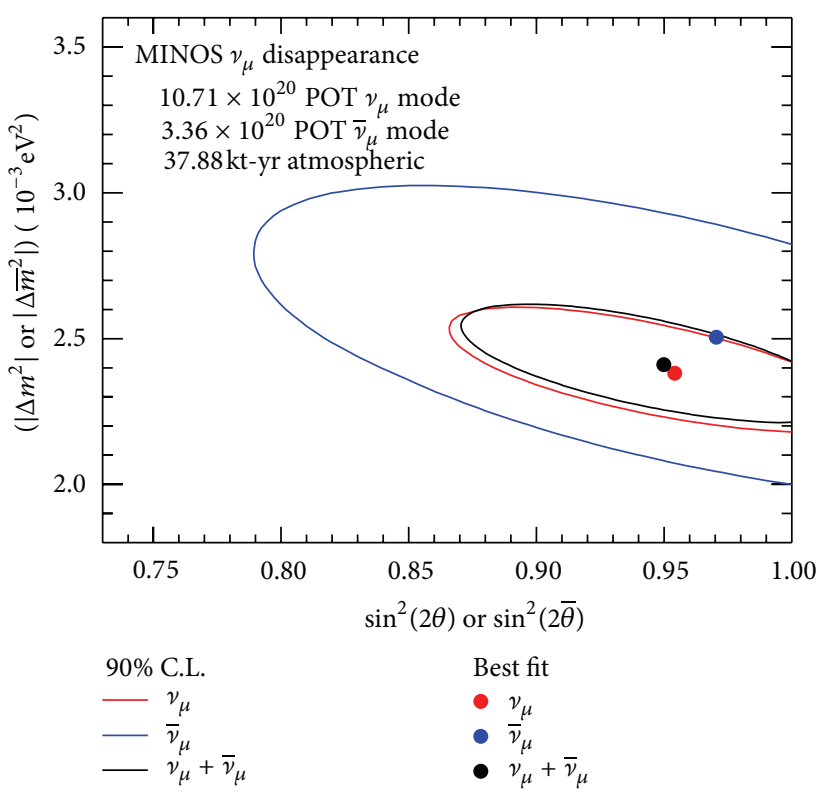

FIGURE 13: The allowed region for antineutrino oscillation parameters (blue line), compared to the region measured with neutrinos alone (red line) and the region measured using both neutrinos and antineutrinos under the assumption they have the same parameters (black line).

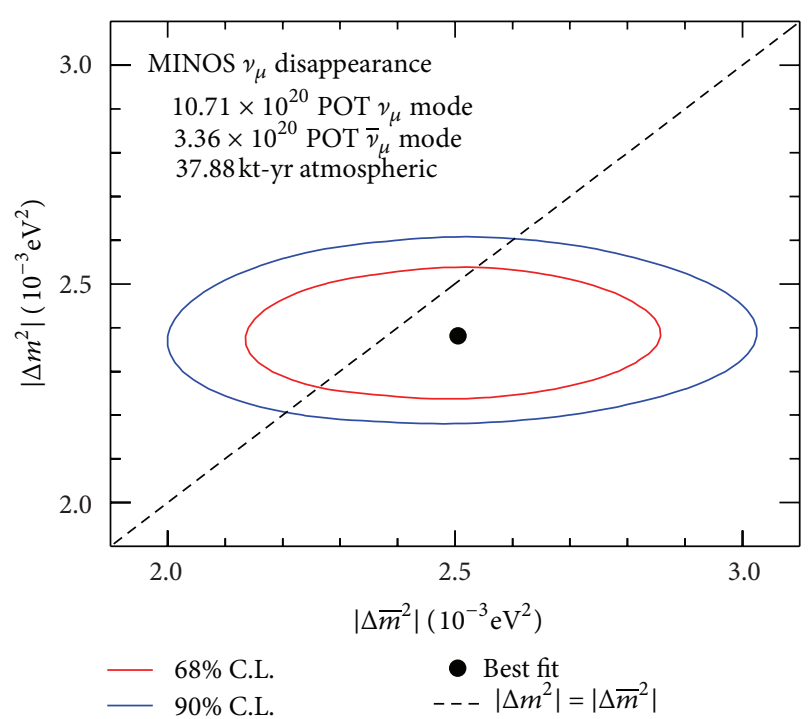

Figure 14: A comparison of the measured limits on the mass splittings of neutrinos and antineutrinos.

the Earth's crust. Assuming a normal mass hierarchy, $\delta=0$, and $\theta_{23}<\pi / 4$, MINOS measures $2 \sin ^{2}\left(2 \theta_{13}\right) \sin ^{2}\left(\theta_{23}\right)=$ $0.051_{-0.030}^{+0.038}$. Assuming an inverted mass hierarchy, $\delta=0$, and $\theta_{23}<\pi / 4$, MINOS measures $2 \sin ^{2}\left(2 \theta_{13}\right) \sin ^{2}\left(\theta_{23}\right)=$ $0.093_{-0.049}^{+0.054}$. This measurement is consistent with the results from reactor neutrino searches [72-74] and the T2K experiment [75].

This MINOS measurement is the first ever search for $\bar{\nu}_{e}$ appearance in a long-baseline $\bar{\nu}_{\mu}$ beam and the first search 


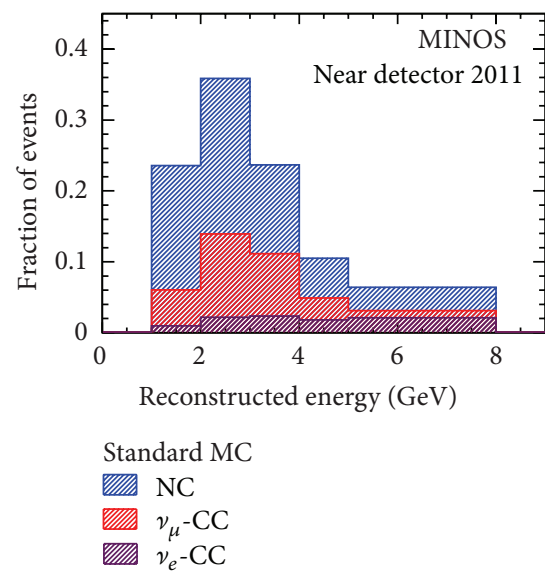

(a)
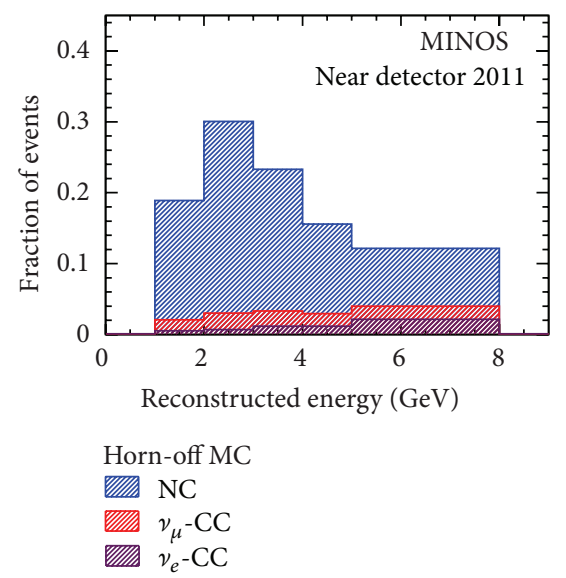

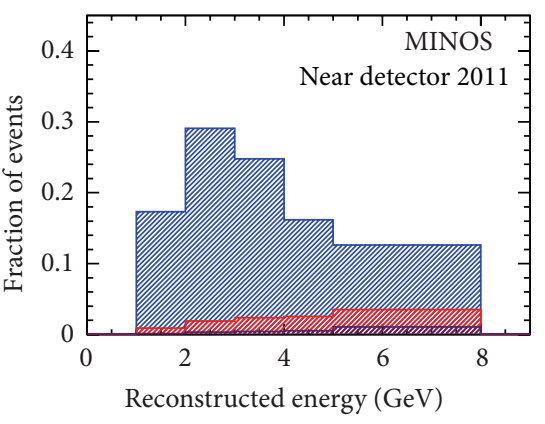

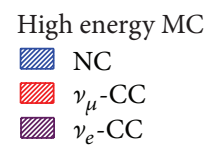

(c)

FIGURE 15: The contribution of the three components to the background in the $v_{e}$ appearance search, as simulated in the ND. (a) the regular (low energy) beam data; (b) with no current in the NuMI focusing horns; (c) a high energy beam configuration.

for $\nu_{e}$ and $\bar{\nu}_{e}$ appearance with significant matter effects. Both of these effects provide some sensitivity to the neutrino mass hierarchy and $\mathscr{C} \mathscr{P}$ violation when the MINOS data is compared to the measurements made by reactor neutrino searches [72-74] (we have calculated a limit of $\sin ^{2}\left(2 \theta_{13}\right)=$ $0.098 \pm 0.013$ from the reactor data at the time of analysis). The sensitivity of MINOS to the mass hierarchy and $\mathscr{C} \mathscr{P}$ violation is modest, but this contributes to the first analysis of the type that will be used by all future long-baseline experiments. The resulting values of the likelihood by which MINOS disfavours various values of these parameters are shown in Figure 18 [76].

\section{Search for Sterile Neutrino Mixing Using Neutral-Current Interactions}

The energy spectrum of NC interactions in the FD should be unchanged by standard neutrino oscillation. The existence of one or more sterile neutrino flavours, $v_{s}$, could cause a deficit in the observed NC interaction rate. As with all the MINOS oscillation analyses, the energy spectrum of NC interactions observed in the ND (which was shown in Figure 8) is used to predict the spectrum expected at the FD [77]. The FD expectation is shown in Figure 19, with the dashed blue line taking into account $v_{e}$ appearance corresponding to $\theta_{13}=$ $11.5^{\circ}$ (at the limit set by CHOOZ [78] and a little above the current accepted value $[29,72-75]$; this analysis uses only the first $7.07 \times 10^{20}$ protons on target of data, and was performed before the recent positive measurements of a nonzero $\left.\theta_{13}\right)$. The data are also shown in the figure and are in good agreement with the expectation, confirming the standard model of neutrino oscillation. This agreement can be quantified using a test statistic $R$ :

$$
R=\frac{N_{\text {data }}-B_{\mathrm{CC}}}{S_{\mathrm{NC}}},
$$

where $N_{\text {data }}$ is the number of events observed, $B_{\mathrm{CC}}$ is the predicted background of CC interactions, and $S_{\mathrm{NC}}$ is the predicted number of $\mathrm{NC}$ interactions. A value of $R=1.01 \pm$ 0.06 (stat.) \pm 0.05 (syst.) is obtained (over the full energy range $0-120 \mathrm{GeV}$ ), which is in good agreement with the expectation of $R=1$ in the case of no mixing with sterile neutrinos.

The data are analysed with a model that assumes a single sterile neutrino flavour, mixing through the addition of a fourth neutrino mass state $m_{4} \gg m_{3}$. This introduces a mass splitting $\Delta m_{43}^{2}$ with magnitude $\mathcal{O}\left(1 \mathrm{eV}^{2}\right)$, such that no oscillation-induced change to the event rate is observed at the $\mathrm{ND}$, and the oscillatory energy dependence of the induced depletion at the FD is so rapid that an overall uniform depletion is observed once the energy resolution of the detectors is accounted for. This model introduces three additional mixing angles, $\theta_{14}, \theta_{24}$, and $\theta_{34}$. MINOS is insensitive to $\theta_{14}$ but sets limits of $\theta_{24}=\left(0.0_{-0.0}^{+5}\right)^{\circ}$ and $\theta_{34}=\left(0.0_{-0.0}^{+25}\right)^{\circ}$. These limits are evaluated assuming $\theta_{13}$ at the $\mathrm{CHOOZ}$ limit.

The limit on the coupling of sterile to active neutrinos can be quantified by defining $f_{s}$, the fraction of disappearing $\nu_{\mu}$ which have oscillated into $v_{s}$ :

$$
f_{s}=\frac{P_{v_{\mu} \rightarrow v_{s}}}{1-P_{v_{\mu} \rightarrow v_{\mu}}} \text {. }
$$

For the model used in which $m_{4} \gg m_{3}, f_{s}$ is evaluated at $1.4 \mathrm{GeV}$ : the energy of maximal $\nu_{\mu}$ disappearance. To determine the limit on $f_{s}$, a large number of test values are chosen of the mixing angles $\theta_{24}, \theta_{34}$, and $\theta_{23}$, from Gaussian distributions according to the measured values and $1 \sigma$ uncertainties given above (with $\theta_{23}$ constrained from the measurements with CC $\nu_{\mu}$ interactions). $f_{s}$ is calculated for each case, and the value of $f_{s}$ that is larger than $90 \%$ of the test cases represents the limit. MINOS limits $f_{s}<0.40$ at the $90 \%$ confidence level.

\section{The Future: MINOS+}

The MINOS experiment has made some very important contributions to our understanding of neutrino oscillation 

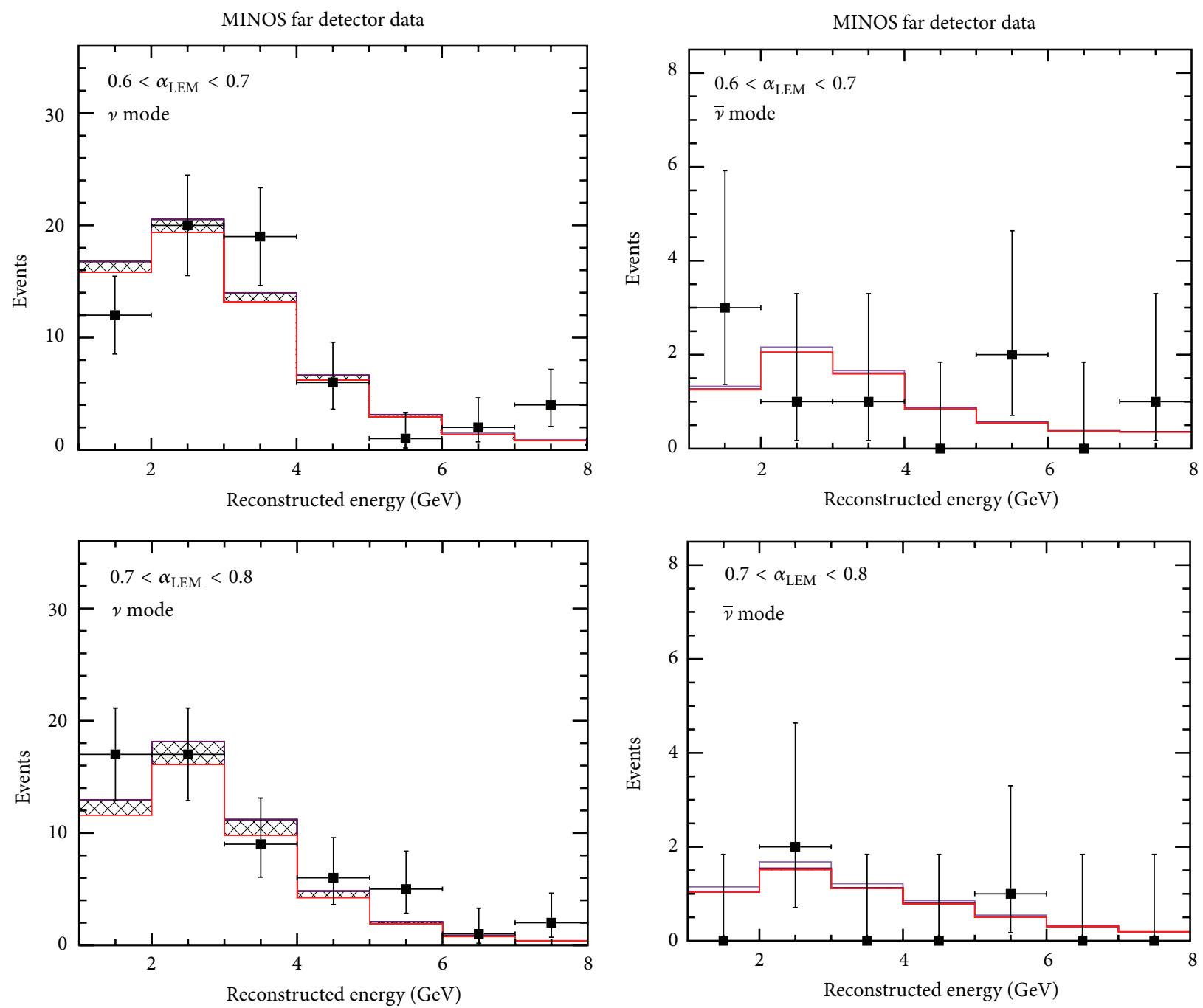

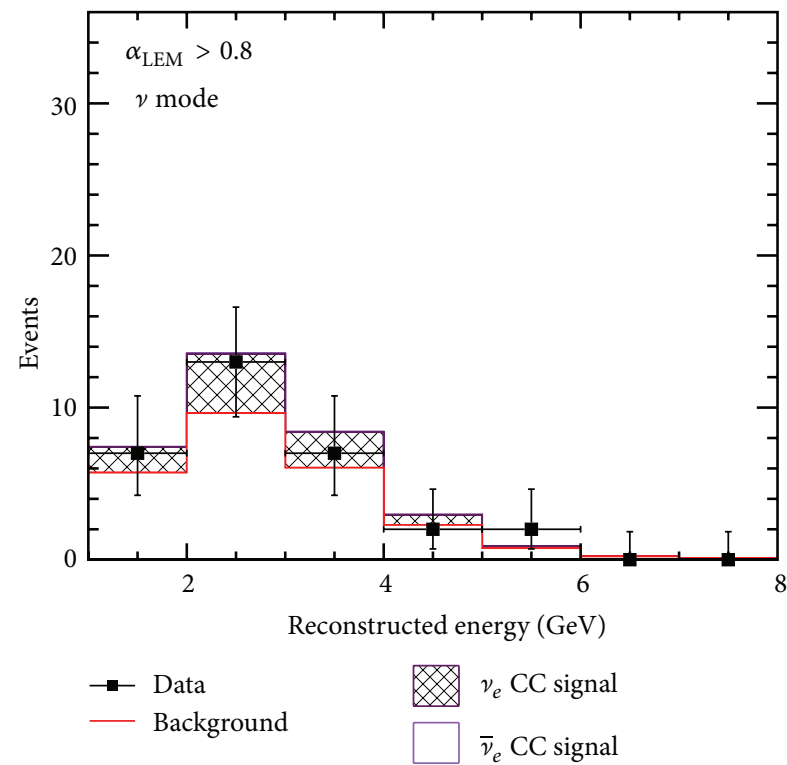

(a)

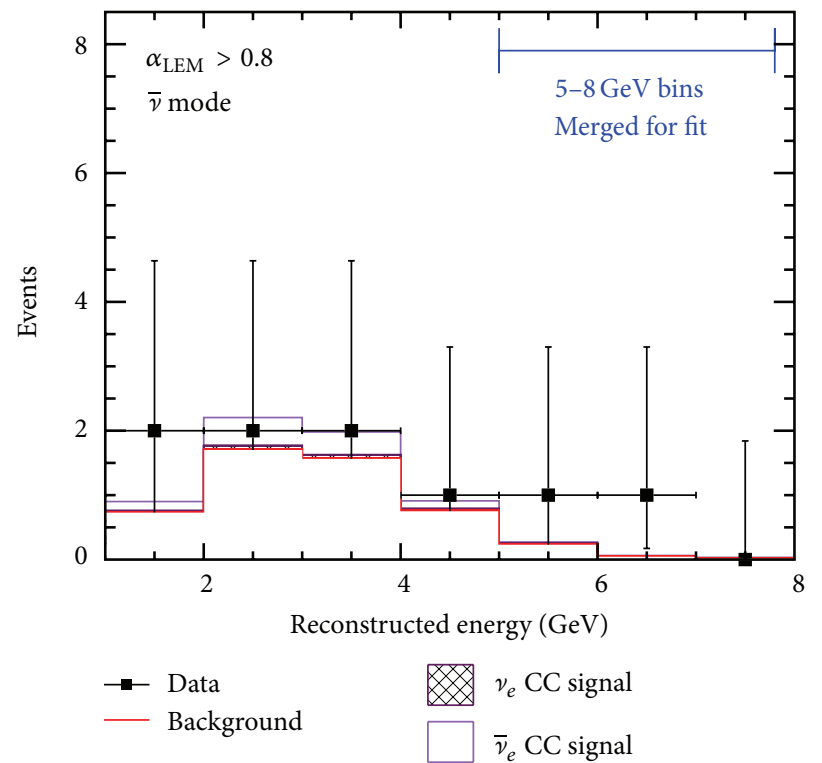

(b)

FIGURE 16: The CC $v_{e}$ (a) and $\bar{\nu}_{e}$ (b) candidate events selected in the FD, compared to the expectation without any $v_{e}$ appearance (red) and with the best fit for $\theta_{13}$ (purple). The events are divided into bins of the library event matching discriminant variable. 


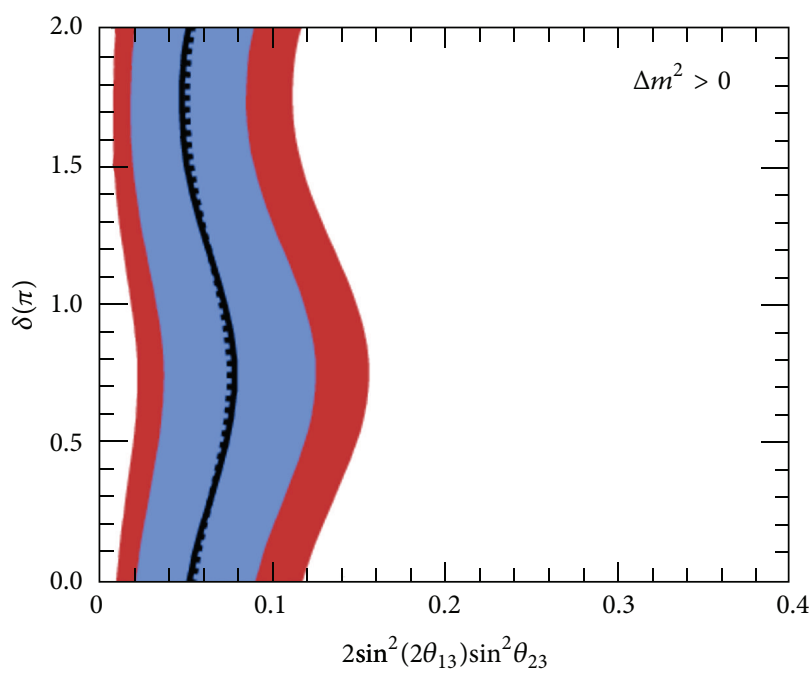

(a)

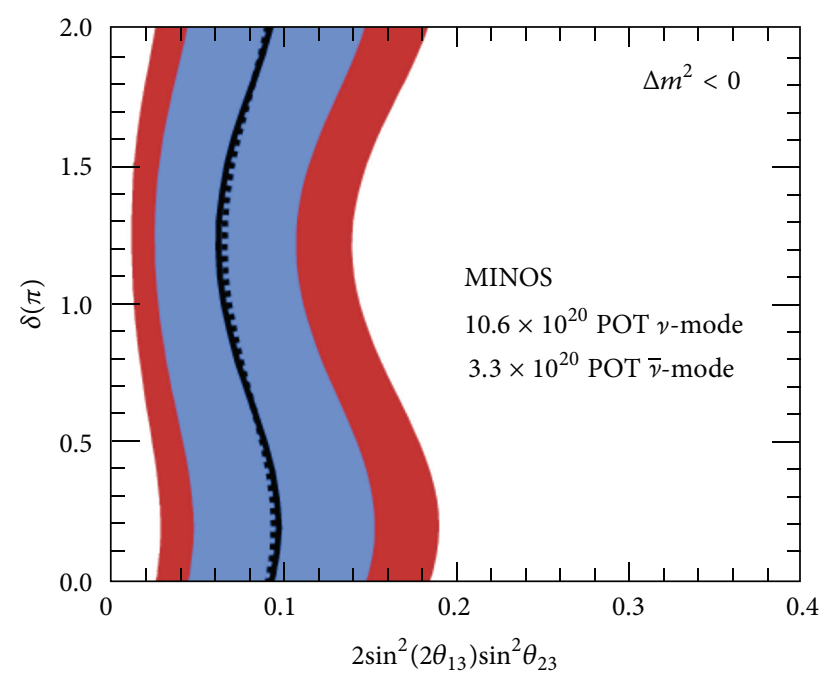

- MINOS best fit $\theta_{23}<\pi / 4$

$68 \%$ C.L. $\theta_{23}<\pi / 4$ $90 \%$ C.L. $\theta_{23}<\pi / 4$

(b)

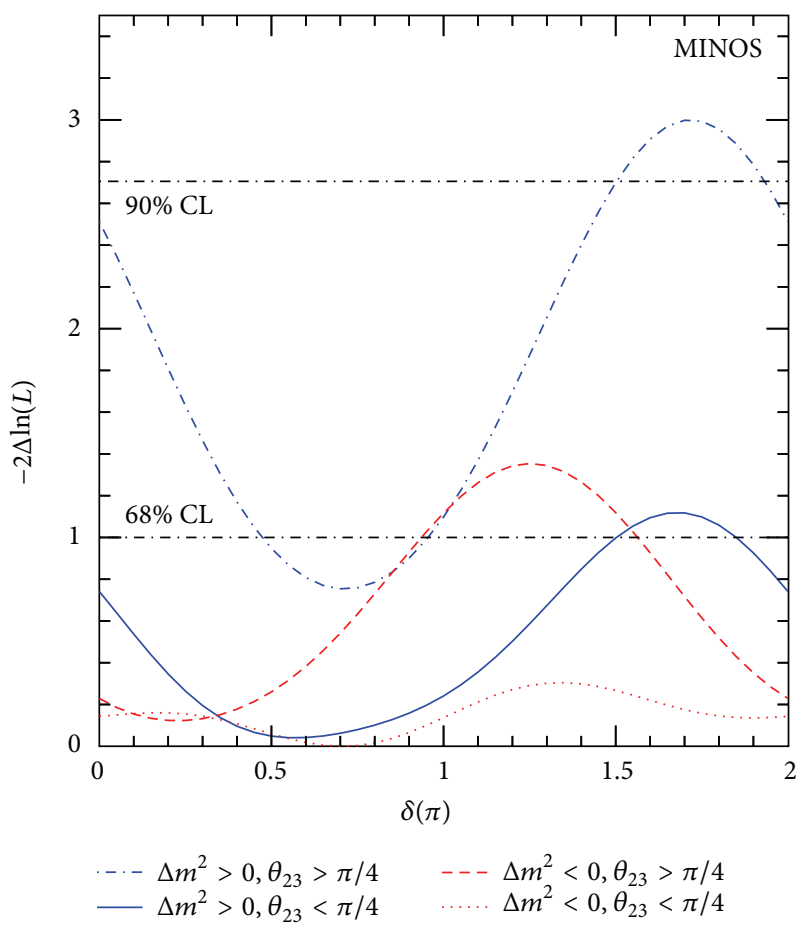

FIGURE 18: The values of likelihood $L$ by which various values of the $\mathscr{C} \mathscr{P}$ violating parameter $\delta$, the mass hierarchy, and the octant of $\theta_{23}$ are disfavoured. This analysis uses MINOS data and information from reactor neutrino measurements of $\theta_{13}$ [72-74].

physics and has finished taking data with the low energy beam for which it was designed. However, the experiment will continue taking data and producing new results for the next few years as MINOS+ [79]. The NuMI beam is being

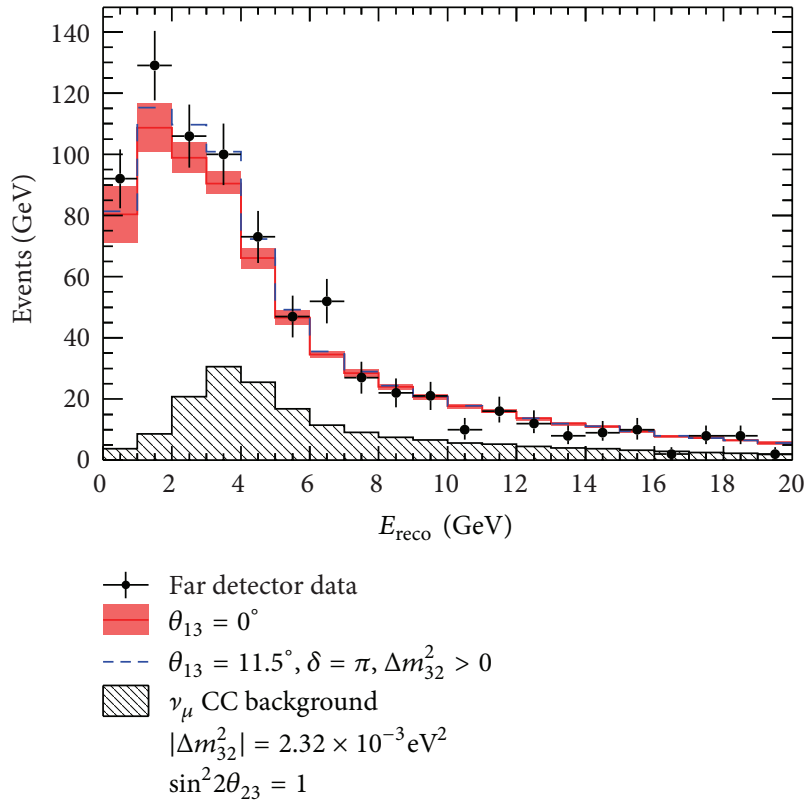

Figure 19: The black dots show the energy spectrum of NC interactions observed in the far detector. The red lines show the expectation in the case of no sterile neutrinos and $\theta_{13}=0$; the blue dashed line shows the same expectation with $\theta_{13}=11.5^{\circ}$.

upgraded to a higher energy and intensity for the $\mathrm{NO} v \mathrm{~A}$ experiment, the far detector of which will sit 14 mrad offaxis. $\mathrm{NO} v \mathrm{~A}$ will receive a narrow-band beam, peaking at around $2 \mathrm{GeV}$, which is ideal for searching for $v_{e}$ appearance since the background seen in MINOS from NC interactions of high energy neutrinos will be heavily reduced. Figure 20 shows that the MINOS FD will see an intense $\nu_{\mu}$ beam, 


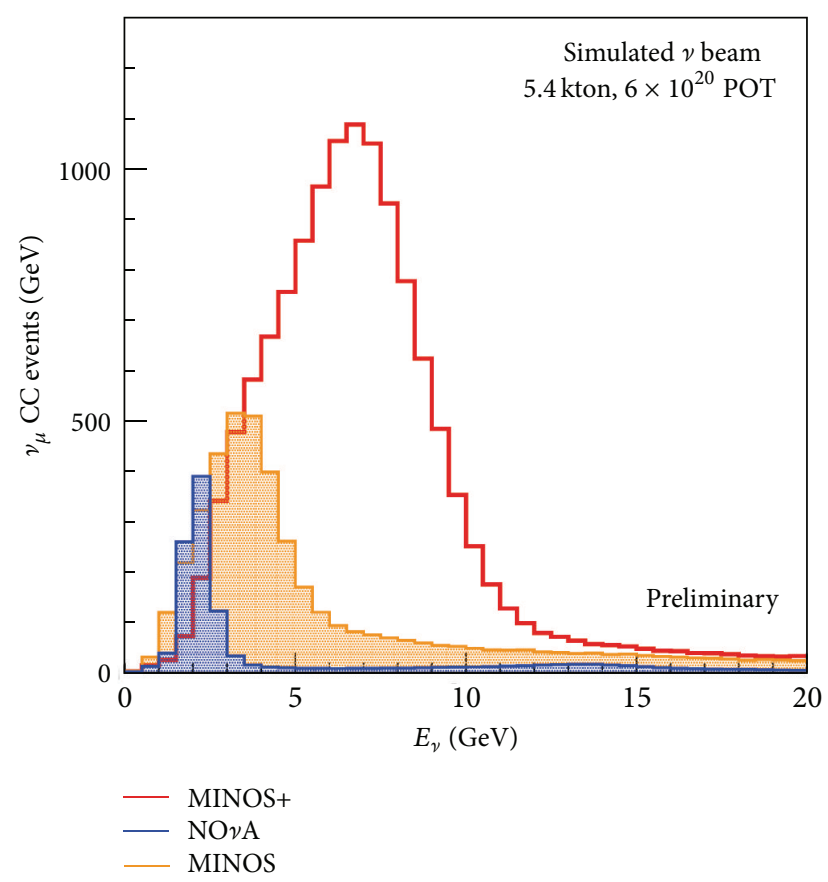

FIgURE 20: The $v_{\mu}$ energy spectrum that will be observed by the MINOS+ FD, compared to the spectra observed by MINOS and $\mathrm{NO} v \mathrm{~A}$.

peaking at around $7 \mathrm{GeV}$. In this configuration, MINOS+ will observe around 4,000 CC $\nu_{\mu}$ interactions in the FD each year, unprecedented statistics for a long-baseline oscillation experiment. This will offer a unique, high precision test of the three-flavour oscillation paradigm.

MINOS+ will be able to make a very sensitive search for the sterile neutrinos suggested by the LSND [80] and MiniBooNE [81, 82] data and by some interpretations of reactor neutrino data [83]. This search will cover more than three orders of magnitude in the mass splitting between the sterile and active neutrinos. The signal that MINOS+ will search for is illustrated in Figure 21. Figure 21(a) illustrates the increase in statistical precision that MINOS+ will provide on the ratio of the observed CC $\nu_{\mu}$ interaction rate to that expected without oscillations. Figure 21(b) shows how this ratio is modified if a sterile neutrino exists and mixes with the active neutrinos: an additional deficit of muon neutrino interactions occurs in the higher energy region of the spectrum. In the model assumed here, which introduces a single sterile neutrino state, three new mixing angles are introduced: $\theta_{14}, \theta_{24}$, and $\theta_{34}$. An additional mass splitting $\Delta m_{43}^{2}$ is also introduced. The position in energy of the additional deficit is governed by the value of the new mass splitting. The magnitude of the additional deficit in the CC $\nu_{\mu}$ interaction rate is governed primarily by the size of $\theta_{24}$; this is in comparison to short-baseline searches for $\bar{\nu}_{e}$ disappearance from reactors which are governed by $\theta_{14}$ and searches for $\nu_{e}$ appearance in $\bar{\nu}_{\mu}$ beams which are governed by a combination of $\theta_{14}$ and $\theta_{24}$. A detailed explanation of the global effort to search for sterile neutrinos can be found in [84].

The sensitivity of the MINOS+ experiment, when combined with the Bugey reactor neutrino data [85], is shown in

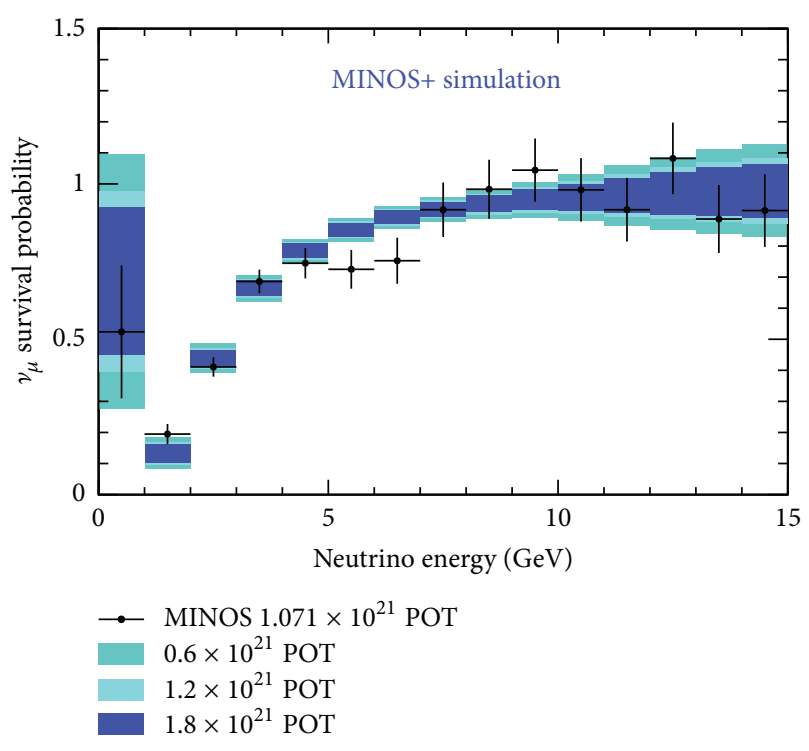

(a)

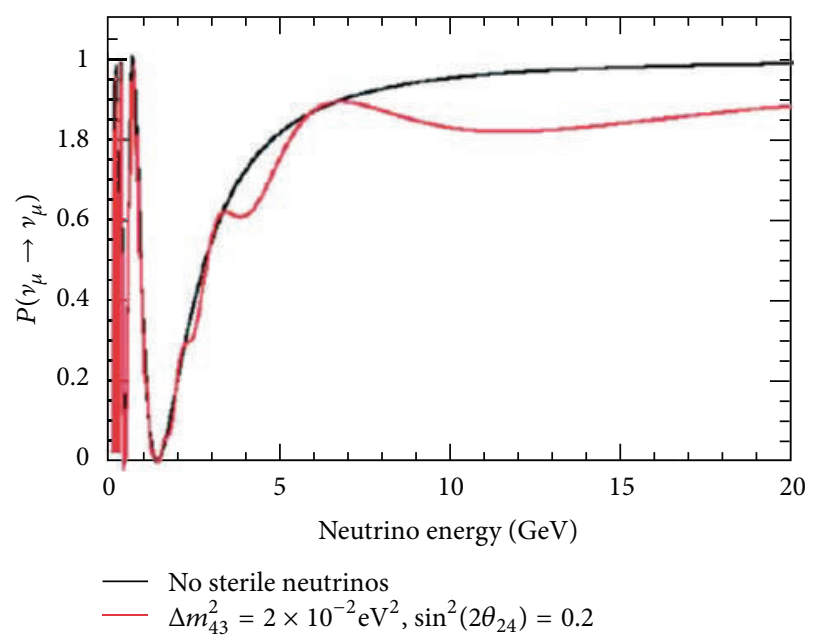

(b)

FIgURE 21: (a) The black points show the ratio of the observed $v_{\mu}$ energy spectrum to the expectation in the case of no oscillation in the neutrino-dominated beam of MINOS. The blue region shows the statistical precision expected from MINOS+. (b) The black line shows the muon neutrino survival probability in the case of no sterile neutrinos; the red line shows how the probability would be modified by the addition of mixing with a sterile neutrino.

Figure 22; MINOS+ has the potential to rule out much of the LSND allowed region. MINOS+ will begin taking data in the late summer of 2013, and will continue taking data for at least two years (Figure 22 assumes two years of data taking with a neutrino-dominated beam).

\section{Conclusion}

The MINOS experiment was conceived at a time when neutrino oscillation had only recently been confirmed as the solution to the problem of neutrino flavour change. It has 


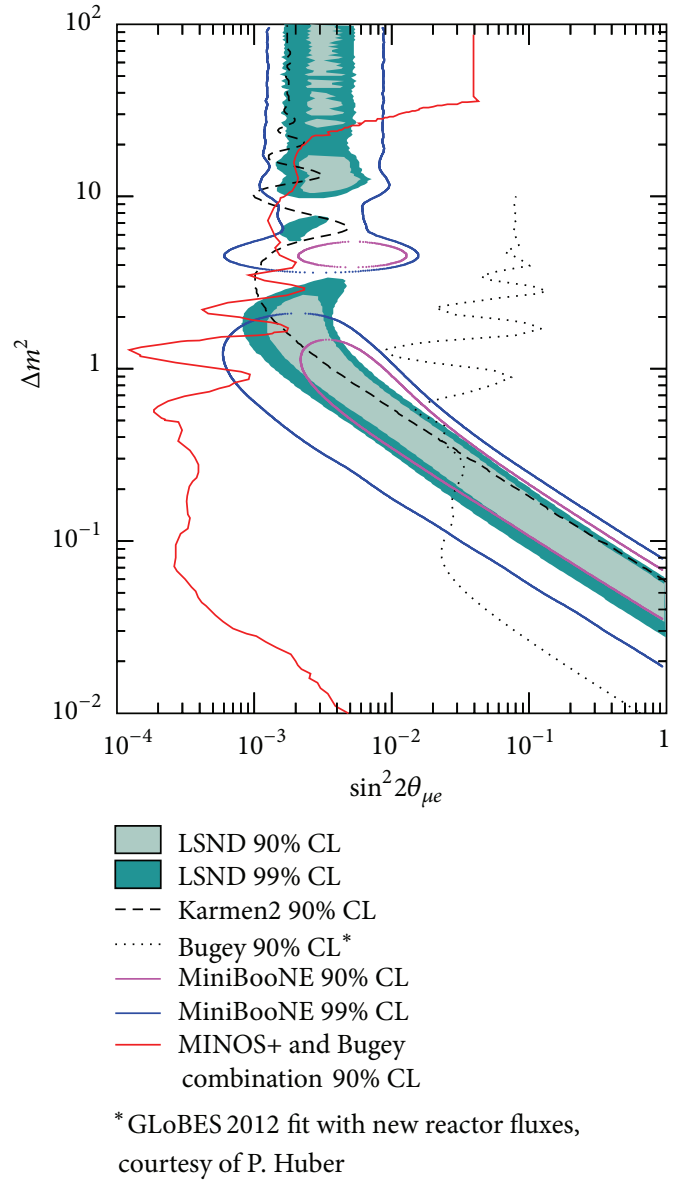

FIGURE 22: The sensitivity of MINOS+ to the existence of sterile neutrinos, when combined with data from the Bugey [85] reactor neutrino experiment. $\Delta m^{2}$ is the splitting between the three known neutrino mass states and a new, fourth state. $\theta_{\mu e}$ is the mixing angle governing $\nu_{\mu} \rightarrow v_{e}$ transitions when a fourth, sterile neutrino state is introduced into the PMNS mixing matrix. This figure assumes two years of MINOS+ running with a neutrino-dominated beam.

played a hugely influential role in bringing neutrino oscillation physics into an era of precision measurement. MINOS's measurement of the largest neutrino mass splitting is the most precise in the world. MINOS has made the first direct precision measurement of the corresponding antineutrino parameters, a measurement that promises to remain the world's most precise for many years. And MINOS has played a role in the discovery of a nonzero value for $\theta_{13}$.

Now that the value of $\theta_{13}$ is known, the neutrino physics community can move on to determine the neutrino mass hierarchy and to search for $\mathscr{C} \mathscr{P}$ violation in the neutrino sector. MINOS has pioneered a number of techniques that will be used by future experiments. The two-detector setup, all important in reducing the impact of systematic uncertainties, is the design of choice for any new experiment, and MINOS has demonstrated methods of using a near detector to determine the expectation at a far detector. MINOS has performed the first search for $\bar{\nu}_{e}$ appearance in a $\bar{v}_{\mu}$ beam and the first search for $\nu_{e}$ and $\bar{\nu}_{e}$ appearance with significant matter effects, demonstrating the analysis techniques that will be used to determine the mass hierarchy and $\mathscr{C} \mathscr{P}$ violation parameter.

In the second half of 2013, MINOS will begin taking data as the MINOS+ experiment, which will make ever more precise tests of the three-flavour neutrino oscillation paradigm and set world-leading limits on the existence of sterile neutrinos. This is an exciting future for an experiment that, with a decade of data taking so far, has already created a lasting legacy for itself in our understanding of the neutrino.

A comparison of the measured neutrino and antineutrino mass splittings is shown in Figure 14; the difference between the two is constrained to $\left|\Delta \bar{m}^{2}\right|-\left|\Delta m^{2}\right|=\left(0.12_{-0.26}^{+0.24}\right) \times$ $10^{-3} \mathrm{eV}^{2}$.

\section{Acknowledgment}

The work of the MINOS and MINOS+ collaborations is supported by the US DoE, the UK STFC, the US NSF, the State and University of Minnesota, the University of Athens in Greece, and Brazil's FAPESP and CNPq. The authors are grateful to the Minnesota Department of Natural Resources, the crew of the Soudan Underground Laboratory, and the personnel of Fermilab, for their vital contributions.

\section{References}

[1] S. Wojcicki, "Long baseline neutrino oscillation programe in the United States," Nuclear Physics B, vol. 77, no. 1-3, pp. 182186, 1999, Proceedings of the 18th International Conference on Neutrino Physics and Astrophysics (Neutrino '98), Takayama, Japan, June 1998.

[2] R. Davis Jr., D. S. Harmer, and K. C. Hoffman, "Search for neutrinos from the sun," Physical Review Letters, vol. 20, no. 21, pp. 1205-1209, 1968.

[3] A. I. Abazov, O. L. Anosov, E. L. Faizov et al., "Search for neutrinos from sun using the reaction Ga-71 (electron-neutrino e-) Ge-71," Physical Review Letters, vol. 67, pp. 3332-3335, 1991.

[4] P. Anselmann, W. Hampel, G. Heusser et al., "Solar neutrinos observed by GALLEX at gran sasso," Physics Letters B, vol. 285, no. 4, pp. 376-389, 1992.

[5] M. Aglietta, G. Battistoni, E. Bellotti et al., "Experimental study of atmospheric neutrino flux in the NUSEX experiment," Europhysics Letters, vol. 8, no. 7, article 611, 1989.

[6] K. S. Hirata, "Observation of a small atmospheric $v_{\mu} / v_{e}$ ratio in Kamiokande," Physics Letters B, vol. 280, no. 1-2, pp. 146-152, 1992.

[7] R. Becker-Szendy, C. B. Bratton, D. Casper et al., "Electronand muon-neutrino content of the atmospheric flux," Physical Review D, vol. 46, pp. 3720-3724, 1992.

[8] K. Daum, W. Rhode, P. Bareyre et al., "Determination of the atmospheric neutrino spectra with the fréjus detector," Zeitschrift für Physik C, vol. 66, no. 3, pp. 417-428, 1995.

[9] S. Ahlen, M. Ambrosio, R. Antolini, and G. Auriemma, "Atmospheric neutrino flux measurement using upgoing muons," Physics Letters B, vol. 357, no. 3, pp. 481-486, 1995.

[10] W. M. Allison, G. J. Alner, D. S. Ayres et al., "Measurement of the atmospheric neutrino flavour composition in Soudan 2," Physics Letters B, vol. 391, no. 3-4, pp. 491-500, 1997. 
[11] Y. Fukuda, T. Hayakawa, E. Ichihara et al., "Evidence for oscillation of atmospheric neutrinos," Physical Review Letters, vol. 81, no. 8, pp. 1562-1567, 1998.

[12] Q. R. Ahmad, R. C. Allen, T. C. Andersen et al., "Measurement of charged current interactions produced by solar neutrinos at the sudbury neutrino observatory," Physical Review Letters, vol. 87, Article ID 071301, 2001.

[13] Q. R. Ahmad, R. C. Allen, T. C. Andersen et al., "Direct evidence for neutrino flavor transformation from neutral-current interactions in the sudbury neutrino observatory," Physical Review Letters, vol. 89, Article ID 011301, 6 pages, 2002.

[14] B. Pontecorvo, "Inverse beta processes and nonconservation of lepton charge," Journal of Experimental and Theoretical Physics, vol. 34, pp. 172-173, 1958.

[15] V. N. Gribov and B. Pontecorvo, "Neutrino astronomy and lepton charge," Physics Letters B, vol. 28, pp. 493-496, 1969.

[16] Z. Maki, M. Nakagawa, and S. Sakata, "Remarks on the unified model of elementary particles," Progress of Theoretical Physics, vol. 28, no. 5, pp. 870-880, 1962.

[17] P. Adamson, C. Andreopoulos, D. J. Auty et al., "First direct observation of muon antineutrino disappearance," Physical Review Letters, vol. 107, no. 2, Article ID 021801, 2011.

[18] P. Adamson, D. J. Auty, D. S. Ayres et al., "Search for the disappearance of muon antineutrinos in the NuMI neutrino beam," Physical Review D, vol. 84, no. 7, Article ID 071103, 6 pages, 2011.

[19] P. Adamson, D. S. Ayres, C. Backhouse et al., "Improved measurement of muon antineutrino disappearance in MINOS," Physical Review Letters, vol. 108, no. 19, Article ID 191801, 5 pages, 2012.

[20] P. Adamson, I. Anghel, C. Backhouse et al., "Measurement of Neutrino and Antineutrino Oscillations Using Beam and Atmospheric Data in MINOS," Physical Review Letters, vol. 110, no. 25, Article ID 251801, 6 pages, 2013.

[21] K. Anderson, B. Bernstein, D. Boehnlein et al., "The NuMI Facility Technical Design Report,” FERMILAB-DESIGN-199801, 1998.

[22] D. G. Michael, P. Adamson, T. Alexopoulos et al., "Observation of muon neutrino disappearance with the MINOS detectors in the NuMI neutrino beam," Physical Review Letters, vol. 97, no. 19, Article ID 191801, 6 pages, 2006.

[23] P. Adamson, C. Andreopoulos, K. E. Arms et al., "Study of muon neutrino disappearance using the fermilab main injector neutrino beam," Physical Review D, vol. 77, no. 7, Article ID 072002, 34 pages, 2008.

[24] P. Adamson, C. Andreopoulos, K. E. Arms et al., "Measurement of neutrino oscillations with the MINOS detectors in the NuMI beam," Physical Review Letters, vol. 101, no. 13, Article ID 131802, 5 pages, 2008.

[25] P. Adamson, C. Andreopoulos, R. Armstrong et al., "Measurement of the neutrino mass splitting and flavor mixing by MINOS," Physical Review Letters, vol. 106, no. 18, Article ID 181801, 6 pages, 2011.

[26] P. Adamson, C. Andreopoulos, K. E. Arms et al., "Search for muon-neutrino to electron-neutrino transitions in MINOS," Physical Review Letters, vol. 103, no. 26, Article ID 261802, 5 pages, 2009.

[27] P. Adamson, C. Andreopoulos, D. J. Auty et al., "New constraints on muon-neutrino to electron-neutrino transitions in MINOS," Physical Review D, vol. 82, no. 5, Article ID 051102, 6 pages, 2010.
[28] P. Adamson, D. J. Auty, D. S. Ayres et al., "Improved search for muon-neutrino to electron-neutrino oscillations in MINOS," Physical Review Letters, vol. 107, no. 18, Article ID 181802, 6 pages, 2011.

[29] P. Adamson, I. Anghel, C. Backhouse et al., "Electron neutrino and antineutrino appearance in the full MINOS data sample," Physical Review Letters, vol. 110, no. 17, Article ID 171801, 6 pages, 2013.

[30] P. Adamson, C. Andreopoulos, K. E. Arms et al., "Search for active neutrino disappearance using neutral-current interactions in the MINOS long-baseline experiment," Physical Review Letters, vol. 101, no. 22, Article ID 221804, 5 pages, 2008.

[31] P. Adamson, C. Andreopoulos, D. J. Auty et al., "Search for sterile neutrino mixing in the MINOS long-baseline experiment," Physical Review D, vol. 81, no. 5, Article ID 052004, 18 pages, 2010.

[32] P. Adamson, D. J. Auty, D. S. Ayres et al., "Active to sterile neutrino mixing limits from neutral-current interactions in MINOS," Physical Review Letters, vol. 107, no. 1, Article ID 011802, 5 pages, 2011.

[33] Ž. Pavlovic, Observation of disappearance of muon neutrinos in the NuMI beam [Ph.D. thesis], University of Texas at Austin, Austin, Tex, USA, 2008.

[34] F. Ballarini, G. Battistoni, M. Campanella et al., "The FLUKA code: an overview," Journal of Physics, vol. 41, article 151, 2006.

[35] S. Agostinelli, J. Allison, K. Amako et al., "GEANT4-a simulation toolkit," Nuclear Instruments and Methods in Physics Research A, vol. 506, no. 3, pp. 250-303, 2003.

[36] G. Battistoni, F. Cerutti, A. Fassò et al., "The FLUKA code: description and benchmarking," in Proceedings of the Hadronic Shower Simulation Workshop, vol. 896 of AIP Conference Proceedings, pp. 31-49, Batavia, Ill, USA, September 2006.

[37] D. G. Michaele, P. Adamson, T. Alexopoulos et al., "The magnetized steel and scintillator calorimeters of the MINOS experiment," Nuclear Instruments and Methods in Physics Research A, vol. 596, no. 2, pp. 190-228, 2008.

[38] I. E. Stockdale, A. Bodek, F. Borcherding, N. Giokaris et al., "Limits on muon-neutrino oscillations in the mass range $30<$ $\triangle m^{2}<1000 \mathrm{eV}^{2} / c^{4}$," Physical Review Letters, vol. 52, no. 16, pp. $1384-1388,1984$.

[39] F. Dydak, G. J. Feldman, C. Guyot et al., "A search for $v_{\mu}$ oscillations in the $\Delta m^{2}$ range $0.3-90 \mathrm{eV}^{2}$," Physics Letters $B$, vol. 134, no. 3-4, pp. 281-286, 1984.

[40] F. Bergsma, J. Dorenbosch, M. Jonker et al., "A search for oscillations of muon neutrinos in an experiment with $L / E \cong 0.7$ km/GeV," Physics Letters B, vol. 142, no. 1-2, pp. 103-110, 1984.

[41] M. H. Ahn, E. Aliu, S. Andringa et al., "Measurement of neutrino oscillation by the K2K experiment," Physical Review D, vol. 74, no. 7, Article ID 072003, 39 pages, 2006.

[42] P. Adamson, T. Alexopoulos, W. W. M. Allison et al., "First observations of separated atmospheric $v_{\mu}$ and $\bar{\nu}_{\mu}$ events in the MINOS detector," Physical Review D, vol. 73, no. 7, Article ID 072002, 2006.

[43] P. Adamson, C. Andreopoulos, K. E. Arms et al., "Chargeseparated atmospheric neutrino-induced muons in the MINOS far detector," Physical Review D, vol. 75, no. 9, Article ID 092003, 14 pages, 2007.

[44] P. Adamson, C. Backhouse, G. Barr et al., "Measurements of atmospheric neutrinos and antineutrinos in the MINOS far detector," Physical Review D, vol. 86, no. 5, Article ID 052007, 20 pages, 2012. 
[45] M. A. Kordosky, Hadronic interactions in the MINOS detectors [Ph.D. thesis], University of Texas at Austin, Austin, Tex, USA, 2004.

[46] P. L. Vahle, Electromagnetic interactions in the MINOS detectors [Ph.D. thesis], University of Texas at Austin, Austin, Tex, USA, 2004.

[47] C. Backhouse, Measuring neutrino oscillation parameters using $v_{\mu}$ disappearance in MINOS [Ph.D. thesis], University of Oxford, Oxford, UK, 2011.

[48] T. M. Cover and P. E. Hart, "Nearest neighbor pattern classification," IEEE Transactions on Information Theory, vol. 13, no. 1, pp. 21-27, 1967.

[49] R. Ospanov, A measurement of muon neutrino disappearance with the MINOS detectors and NuMI beam [Ph.D. thesis], University of Texas at Austin, Austin, Tex, USA, 2008.

[50] J. S. Marshall, A study of muon neutrino disappearance with the MINOS detectors and the NuMI neutrino beam [Ph.D. thesis], University of Cambridge, Cambridge, UK, 2008.

[51] J. P. Ochoa, A search for muon neutrino to electron neutrino oscillations in the MINOS Experiment [Ph.D. thesis], The California Institute of Technology, Pasadena, Calif, USA, 2009.

[52] R. Toner, Measuring $\theta_{13}$ via muon neutrino to electron neutrino oscillations in the MINOS experiment [Ph.D. thesis], University of Cambridge, Cambridge, UK, 2011.

[53] A. Holin, Electron neutrino appearance in the MINOS experiment [Ph.D. thesis], University College London, London, UK, 2010.

[54] J. Boehm, Measurement of electron neutrino appearance with the MINOS experiment [Ph.D. thesis], Harvard University, Cambridge, Mass, USA, 2009.

[55] G. Tinti, Sterile neutrino oscillations in MINOS and hadron production in $p C$ collisions [Ph.D. thesis], University of Oxford, Oxford, UK, 2010.

[56] J. J. Evans, Measuring antineutrino oscillations with the MINOS experiment [Ph.D. thesis], University of Oxford, Oxford, UK, 2008.

[57] S. J. Coleman, A measurement of neutrino oscillations with muon neutrinos in the MINOS experiment [Ph.D. thesis], College of William \& Mary, Williamsburg, Va, USA, 2011.

[58] J. S. Mitchell, Measuring $v_{\mu}$ disappearance with the MINOS experiment [Ph.D. thesis], University of Cambridge, Cambridge, UK, 2011.

[59] A. McGowan, Observation of deficit in NuMI neutrino-induced rock and non-fiducial muons in MINOS far detector and measurement of neutrino oscillation parameters [Ph.D. thesis], University of Minnesota, Minneapolis, Minn, USA, 2007.

[60] M. Strait, Measurement of neutrino oscillation parameters using anti-fiducial charged current events in MINOS [Ph.D. thesis], University of Minnesota, Minneapolis, Minn, USA, 2010.

[61] Y. Itow, "Atmospheric neutrinos-results from running experiments," in Proceedings of the 25th International Conference on Neutrino Physics and Astrophysics (Neutrino '12), Kyoto, Japan, June 2012.

[62] K. Abe, N. Abgrall, Y. Ajima et al., "First muon-neutrino disappearance study with an off-axis beam," Physical Review D, vol. 85, no. 3, Article ID 031103, 8 pages, 2012.

[63] K. Nakamura, "Review of particle physics," Journal of Physics G, vol. 37, Article ID 075021, 2010.

[64] L. Wolfenstein, "Neutrino oscillations in matter," Physical Review D, vol. 17, no. 9, pp. 2369-2374, 1978.
[65] J. W. F. Valle, "Resonant oscillations of massless neutrinos in matter," Physics Letters B, vol. 199, no. 3, pp. 432-436, 1987.

[66] M. C. Gonzalez-Garcia, M. M. Guzzo, P. I. Krastev et al., "Atmospheric neutrino observations and flavor changing interactions," Physical Review Letters, vol. 82, no. 16, pp. 3202-3205, 1999.

[67] A. Friedland, C. Lunardini, and M. Maltoni, "Atmospheric neutrinos as probes of neutrino-matter interactions," Physical Review D, vol. 70, no. 11, Article ID 111301, 2004.

[68] Z. Isvan, Antineutrino oscillations and a Search for non-standard Interactions with the MINOS [Ph.D. thesis], University of Pittsburgh, Pittsburgh, Pa, USA, 2012.

[69] W. A. Mann, D. Cherdack, W. Musial, and T. Kafka, "Apparent multiple $\Delta m_{32}{ }^{2}$ in $\bar{v}_{\mu}$ and $v_{\mu}$ survival oscillations from nonstandard interaction matter effect," Physical Review D, vol. 82, no. 11, Article ID 113010, 8 pages, 2010.

[70] J. Kopp, P. A. N. Machado, and S. J. Parke, "Interpretation of MINOS data in terms of nonstandard neutrino interactions," Physical Review D, vol. 82, no. 11, Article ID 113002, 12 pages, 2010.

[71] J. A. B. Coelho, Investigacao de mecanismos alternativos a oscilacao de neutrinos no experimentos MINOS [Ph.D. thesis], Universidade Estadual de Campinas, São Paulo, Brazil, 2012.

[72] F. P. An, Q. An, J. Z. Bai, A. B. Balantekin et al., "Improved measurement of electron antineutrino disappearance at Daya Bay," Chinese Physics C, vol. 37, Article ID 011001, 21 pages, 2013.

[73] J. K. Ahn, S. Chebotaryov, J. H. Choi et al., "Observation of reactor electron antineutrinos disappearance in the RENO experiment," Physical Review Letters, vol. 108, Article ID 191802, 6 pages, 2012.

[74] Y. Abe, C. Aberle, J. C. dos Anjos et al., "Reactor electron antineutrino disappearance in the Double Chooz experiment," Physical Review D, vol. 86, Article ID 052008, 2012.

[75] K. Abe, N. Abgrall, H. Aihara et al., "Evidence of electron neutrino appearance in a muon neutrino beam," Physical Review D, vol. 88, no. 3, Article ID 032002, 41 pages, 2013.

[76] A. Schreckenberger, Electron neutrino and antineutrino appearance in the MINOS detector [Ph.D. thesis], University of Minnesota, Minneapolis, Minn, USA, 2013.

[77] D. J. Koskinen, MINOS sterile neutrino search [Ph.D. thesis], University College London, London, UK, 2009.

[78] M. Apollonio, A. Baldini, C. Bemporad et al., "Search for neutrino oscillations on a long base-line at the $\mathrm{CHOOZ}$ nuclear power station," The European Physical Journal C, vol. 27, pp. 331374, 2003.

[79] G. Tzanankos, M. Bishai, M. Diwan et al., MINOS+: a proposal to FNAL to run MINOS with the medium energy NuMI beam [Ph.D. thesis], University of Athens, Athens, Greece, 2011.

[80] A. Aguilar, L. B. Auerbach, R. L. Burman et al., "Evidence for neutrino oscillations from the observation of $\bar{\nu}_{e}$ e appearance in a $\bar{\nu}_{\mu}$ beam," Physical Review D, vol. 64, Article ID 112007, 22 pages, 2001.

[81] A. A. Aguilar-Arevalo, C. E. Anderson, S. J. Brice et al., "Search for electron antineutrino appearance at the $\Delta m \sim 1 \mathrm{eV}^{2}$ Scale," Physical Review Letters, vol. 103, no. 11, Article ID 111801, 2009.

[82] A. A. Aguilar-Arevalo, C. E. Anderson, A. O. Bazarko et al., "Improved search for $\bar{v}_{\mu} \rightarrow \bar{v}_{e}$ oscillations in the MiniBooNE experiment," Physical Review Letter, vol. 110, no. 10, Article ID 161801, 2013.

[83] G. Mention, M. Fechner, T. Lasserre et al., "Reactor antineutrino anomaly," Physical Review D, vol. 83, Article ID 073006, 20 pages, 2011. 
[84] K. N. Abazajian, M. A. Acero, S. K. Agarwalla et al., "Light sterile neutrinos: a white paper," http://arxiv.org/abs/1204.5379.

[85] B. Achkar, R. Aleksan, M. Avenier et al., "Search for neutrino oscillations at 15, 40 and 95 meters from a nuclear power reactor at Bugey," Nuclear Physics B, vol. 434, no. 3, pp. 503-532, 1995. 

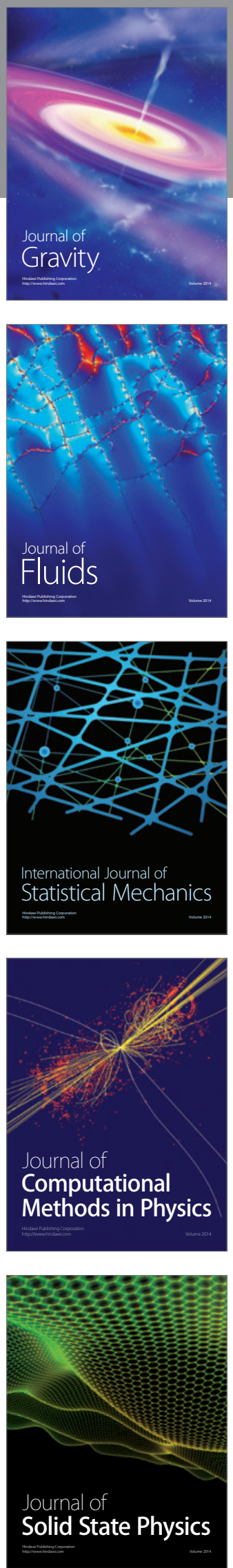

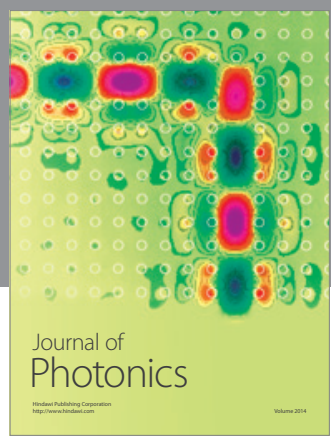

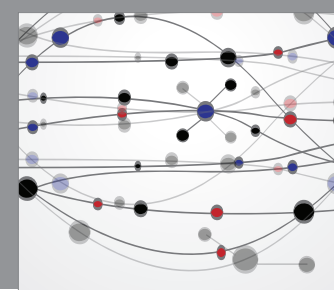

The Scientific World Journal

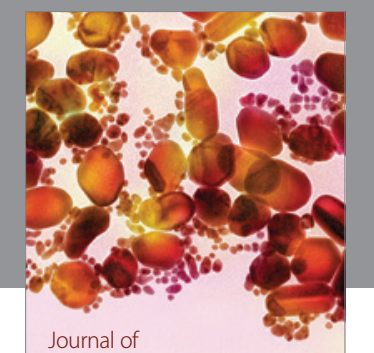

Soft Matter
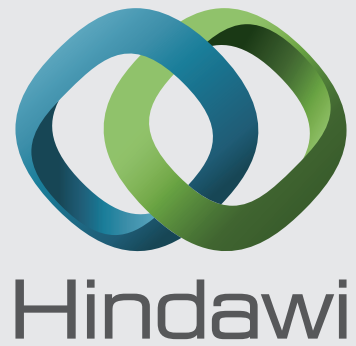

Submit your manuscripts at

http://www.hindawi.com
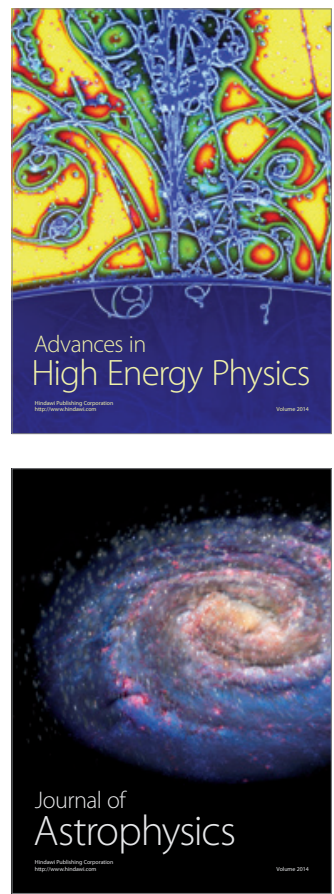
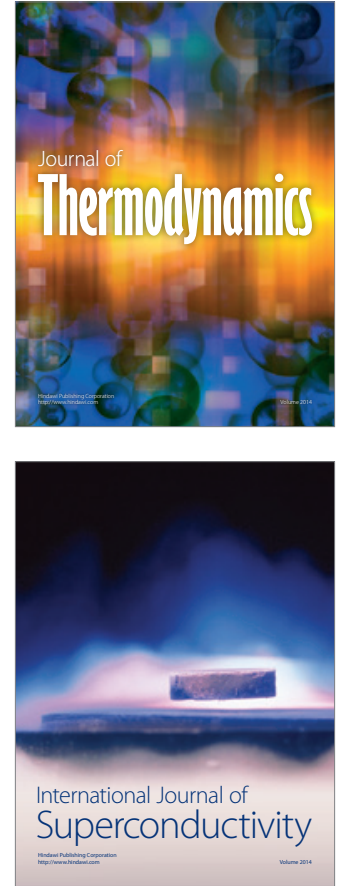
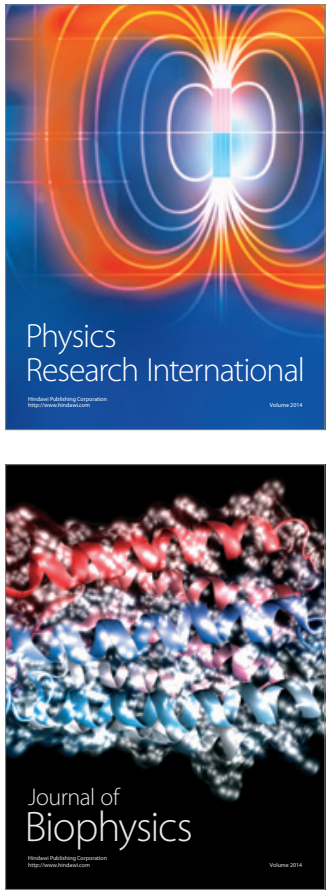
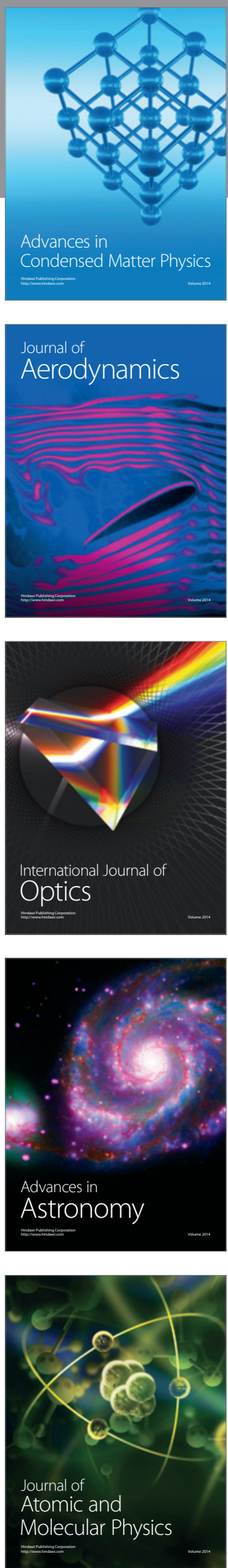